COMMUNICATIONS IN

ANALYSIS AND GEOMETRY

Volume 6, Number 2, 331-392, 1998

\title{
Adiabatic limits of the Seiberg-Witten equations on Seifert manifolds
}

\author{
LIVIU I.NICOLAESCU
}

\section{Introduction.}

The "old" instanton theory naturally lead to the instanton Floer homology of a 3-manifold as the missing piece in a general gluing formula for the Donaldson invariants. Similarly, the Seiberg-Witten theory leads to the "monopole homology" which is the Floer homology of the Seiberg-Witten functional defined by a $\operatorname{spin}^{c}$ manifold (see [KM], [Mar], [MW], [Wa]).

The first main difficulty in understanding this homology comes from the fact that its defining chains are not as explicit as the chains which generate the instanton homology. In the latter case, these are the flat connections on an $S U(2)$ bundle which are well understood both topologically and geometrically. The meaning of the chains in monopole homology is far from obvious and the only explicit computations were made when the 3-manifold $N$ is a product $S^{1} \times \Sigma$ where $\Sigma$ is a surface of genus $\geq 2$ (see [D] or [MST]). The equations are tractable in this case is because $S^{1} \times N$ is a Kähler manifold. As was pointed out in [D], the solutions of the 3D Seiberg-Witten equations coincide with the $S^{1}$-invariant solutions of the $4 \mathrm{D}$ Seiberg Witten equations. Fortunately, on a Kähler manifold the solutions of these equations can be described explicitly.

If now $N$ is the total space of a principal $S^{1}$ bundle of nonzero degree over a surface $\Sigma$ then $S^{1} \times N$ admits a natural complex structure but this time the manifold cannot be Kähler for the simple reason that the first Betti number is odd.

We analyze the Seiberg-Witten equations on a special class of Riemann 3-manifolds, namely those which admit a Killing vector field of constant pointwise length satisfying an additional technical condition. Topologically, these manifolds must be Seifert fibered manifolds.

On such manifolds the Dirac operators have an especially nice form and in particular, the Seiberg-Witten equations can be further dissected. We are 
interested in the behavior of the solutions of the Seiberg-Witten equations as the metric degenerates in the direction of the Killing vector field. This corresponds to collapsing the fibers of the Seifert fibration.

The paper is divided into four parts. The first part describes in detail the differential geometric background. We build our geometry on an anisotropic foundation algebraically encoded as a metric almost contact structure (m.a.c.). This is an $U(1)$-reduction of the tangent frame bundle of our 3-manifold. In particular, we distinguish a special class of such manifolds the so called Killing m.a.c manifolds. These are Riemann manifolds which admit a Killing vector field $\zeta$ of pointwise length 1 . Any oriented Killing m.a.c 3-manifold is diffeomorphic to a Seifert manifold and moreover, any Seifert 3-manifold admits a Killing m.a.c structure. A special class of Killing m.a.c. 3-manifolds consists of the $(K, \lambda)$ manifolds, $\lambda \in \mathbb{R}$. They are characterized by the condition

$$
d \eta(x) \equiv 2 \lambda * \eta
$$

where $\eta$ denotes the 1-form dual to the Killing vector field $\zeta$ and $\lambda$ is a constant. These manifolds are also characterized by the fact that their product with $S^{1}$ admits a natural integrable complex structure.

The total space of a principal $S^{1}$-bundle admits a natural $(K, \lambda)$ structure described for the first time by Boothby and Wang. We present a 1-parameter family of such nice metric structures and we explicitly compute its differential geometric invariants: the Levi-Civita connection, the Ricci and the scalar curvature. Factoring with suitable groups of isometries one can construct many other interesting examples. In particular, in subsection $\S 1.4$ we show that any Seifert manifold admits a natural $(K, \lambda)$-structure. More precisely, the Thurston geometries on Seifert manifolds (from the list of 6 described in $[\mathrm{S}])$ are $(K, \lambda)$ structures. The scalar $\lambda$ is proportional with the Euler number of the Seifert fibration and should be regarded as a measure of "twisting" of the fibration. As the metric degenerates (and so the fibers become shorter and shorter) $\lambda$ will go to zero and thus the fibration will become "less and less twisted".

As was observed by several authors ([ENS], [V]) the $(K, \lambda)$ structures with $\lambda>0$ are links of quasihomogeneous singularities. Their Thurston geometry is (almost) uniquely determined by the analytical structure of the singularity and conversely, (see $[\mathrm{Ne}]$ or $[\mathrm{Sch}]$ ) the Thurston geometry fixes the analytical type of the singularity.

The second part is devoted to Dirac operators on m.a.c manifolds. The spinor bundles corresponding to the various $\operatorname{spin}^{c}$ structures can be very 
nicely described in the almost-contact language. An important part of this section is devoted to a commutator identity which is a key ingredient in the study of adiabatic limits. This is responsible for the adiabatic decoupling of the equations which in the limit look like a pair of equations, one along the fibers and one along the base.

In the third part we introduce the 3-dimensional Seiberg-Witten equations and study the adiabatic limits of solutions as the metric degenerates in the direction of $\zeta$. In particular, we noticed an interesting analytical phenomenon. Although the various Dirac operators "explode" in the adiabatic limit, one can show that their graphs have a very nice behavior. More precisely, they converge (in the gap topology described e.g. in $[\mathrm{K}]$ ). The gap convergence is responsible for uniform estimates for resolvents and, as shown in the works $[\mathrm{MM}]$ and [Dai], these estimates are essential in controlling the behavior of their spectra in the adiabatic limit. We believe this phenomenon of graph convergence is not restricted to three dimensions and deserves further investigation.

On an $S^{1}$ - bundle of degree $\ell$ over a surface of genus $g$ the "adiabatic picture" is similar to the exact descriptions in [D], [MST] or [Mun] when the 3-manifold is the trivial $S^{1}$-bundle over a surface. The set of all possible adiabatic limits consists of two parts: a reducible component (a torus of flat connections) and an irreducible part which is an union of moduli spaces of vortex pairs over the basis of the fibration. According to $[\mathrm{Br}]$, these vortex moduli spaces can be identified with symmetric products of smooth algebraic curves and such objects were analyzed in great detail in [MD]. On an arbitrary Seifert manifold the irreducible part of the adiabatic moduli space consists of abelian vortices on 2-orbifolds. Remarkably, the adiabatic moduli space can be alternatively described as the solution set of an "adiabatic Seiberg-Witten equation" which is a very simple and explicit perturbation of the original one.

The fourth section deals with the variational features of the adiabatic moduli space on a smooth $S^{1}$-fibration over a surface. This moduli space is the critical set of a certain energy functional and its irreducible part is smooth and Bott nondegenerate. Additionally, we provide accurate spectral estimates for the Hessian of this functional on directions normal to the critical set (Corollary 4.8). Two key facts lie behind these estimates. The first is a vanishing result for the deformation complex of the abelian vortex problem on a Riemann surface while the second is a "rigidity" result. More precisely, the Hessian is naturally $S^{1}$-equivariant and the weights of the $S^{1}$-action on its kernel are all trivial. 
The above results suggest that, for topological purposes, the adiabatic Seiberg-Witten equations are better suited for the concrete computations of the Seiberg-Witten-Casson invariant and the Seiberg-Witten-Floer homology.

Note. After this work was completed we learned that similar results on Seiberg-Witten monopoles on Seifert manifolds were independently established in [MOY].

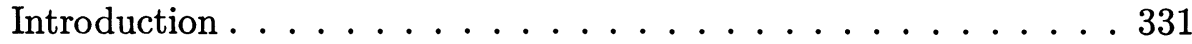

1 Metric almost contact structures . . . . . . . . . . 334

$1.1 \quad$ Basic objects . . . . . . . . . . . . . . 335

1.2 The structural equations of a m.a.c. manifold . . . . 337

1.3 The Boothby-Wang construction . . . . . . . . . . 341

1.4 Geometric Seifert structures . . . . . . . . . . . . . 343

2 Dirac operators on 3-manifolds . . . . . . . . . . 345

2.1 3-dimensional spinorial algebra . . . . . . . . . 345

2.2 3-dimensional spin geometry . . . . . . . . . . 348

2.3 Pseudo Dolbeault operators . . . . . . . . . . . . 349

2.4 Geometric Dirac operators . . . . . . . . . . . . . 355

3 The Seiberg-Witten equations . . . . . . . . . . . . 356

3.1 Generalities . . . . . . . . . . . . . . . . 357

3.2 Adiabatic limits . . . . . . . . . . . . . . 360

3.3 The Seiberg-Witten equations on circle bundles . . . . 367

4 Variational features of the adiabatic moduli space . . . . . . 373

4.1 Algebraic and analytic preliminaries . . . . . . . 373

4.2 Abelian vortices on Riemann surfaces . . . . . . . 378

4.3 Bott nondegeneracy . . . . . . . . . . . . . 382

\section{Metric almost contact structures.}

In this section we describe a differential-geometric setting rich in effects on the Dirac equations in general and the Seiberg-Witten equations in particular. An important feature of such geometries is their anisotropy described as an almost contact structure satisfying additional metric properties. We compute the most important geometric invariants of such structures and then study their behavior under anisotropic adiabatic deformations. This will allow us to conclude that these geometries exist on any (and only on) Seifert manifolds and they are intimately related to Thurston's geometries. 


\subsection{Basic objects.}

Consider an oriented 3-manifold $N$.

Definition 1.1. (a) An almost contact structure on $N$ is a nowhere vanishing 1-form $\eta \in \Omega^{1}(N)$.

(b) A Riemann metric $g$ on $N$ is said to be compatible with an almost contact structure $\eta$ if $|\eta(x)|_{g}=1$ for all $x \in N$. A metric almost contact structure (m.a.c) on $N$ is a pair $(\eta, g)=$ (almost contact structure, compatible metric).

Consider a m.a.c structure $(\eta, g)$ on the oriented 3-manifold $N$. A local, oriented, orthonormal frame $\left\{\zeta_{0}, \zeta_{1}, \zeta_{2}\right\}$ of $T N$ is said to be adapted to the m.a.c. structure if $\zeta_{0}$ is the metric dual of $\eta$. The dual coframe of an adapted frame $\left\{\zeta_{0}, \zeta_{1}, \zeta_{2}\right\}$ has the form $\left\{\eta^{0}, \eta^{1}, \eta^{2}\right\}$ where $\eta^{0}=\eta$ and $* \eta=\eta^{1} \wedge \eta^{2}$. In the sequel we will operate exclusively with adapted frames.

Denote by $C l(N)$ the bundle of Clifford algebras generated by $T^{*} N$ equipped with the induced metric. The quantization map

$$
\text { exterior algebra } \rightarrow \text { Clifford algebra }
$$

(see $[\mathrm{BGV}])$ induces a map

$$
\mathfrak{q}: \Lambda^{*} T^{*} N \rightarrow C l(N) .
$$

On the other hand $\Lambda^{*} T^{*} N$ has a natural structure of $C l(N)$-module so that via the quantization map we can construct an action of $\Lambda T^{*} M$ on itself

$$
\text { c }: \Lambda^{*} T^{*} N \rightarrow \text { End }\left(\Lambda^{*} T^{*} N\right)
$$

called Clifford multiplication.

On a m.a.c. 3-manifold $(N, \eta, g)$ the Clifford multiplication by $* \eta$ has a remarkable property. More precisely

$$
\mathfrak{c}(* \eta)\langle\eta\rangle^{\perp}=\langle\eta\rangle^{\perp} \subset \Lambda^{*} T^{*} N .
$$

If $\left(\eta^{0}, \eta^{1}, \eta^{2}\right)$ is a local coframe then the bundle $\langle\eta\rangle^{\perp}$ is locally spanned by $\eta^{1}, \eta^{2}$ and $\mathfrak{c}(* \eta)$ acts according to the prescription

$$
\mathfrak{c}(* \eta): \eta^{1} \mapsto \eta^{2}, \eta^{2} \mapsto-\eta^{1} .
$$

In particular, notice that both $\mathfrak{c}(* \eta)$ and $-\mathfrak{c}(* \eta)$ define complex structures on the real 2-plane bundle $\langle\eta\rangle^{\perp}$. 
Definition 1.2. The complex line bundle $\left(\langle\eta\rangle^{\perp},-\mathfrak{c}(* \eta)\right)$ is called the canonical line bundle of the m.a.c. 3-manifold $(N, \eta, g)$ and is denoted by $\mathcal{K}=\mathcal{K}_{\eta, g}$.

When viewed as a real bundle $\mathcal{K}$ (and hence $\mathcal{K}^{-1}$ as well) has a natural orientation. We have an isomorphism of oriented real vector bundles

$$
T^{*} N \cong\langle-\eta\rangle \oplus \mathcal{K} \cong\langle\eta\rangle \oplus \mathcal{K}^{-1}
$$

where $\langle-\eta\rangle$ (resp $\langle\eta\rangle)$ denotes the real line bundle spanned and oriented by $-\eta($ resp $\eta)$.

We will be interested only in a special type of m.a.c. structures. More specifically, we will consider only Killing m.a.c manifolds. A m.a.c. structure $(g, \eta, \zeta)$ will be called Killing if the vector field $\zeta$ is Killing. This geometric condition has substantial impact on the topology of the background manifold. More precisely, we have the following result.

Proposition 1.3. Assume $N$ is a compact oriented Killing m.a.c 3-manifold. Then $N$ is diffeomorphic to an oriented Seifert fibered 3-manifold. Conversely, any compact oriented Seifert 3-manifold admits a Killing m.a.c structure.

Proof. Suppose $N$ is Killing m.a.c. Then the collection of Killing m.a.c. structures $(g, \eta)$ with respect to the fixed metric $g$ are parameterized by the unit sphere in the Lie algebra of compact Lie group of isometries Isom $(N, g)$. In particular, the group Isom $(N, g)$ has positive dimension. If this is the case, the maximal torus containing $\zeta$ induces at least one fixed-point-free $S^{1}$ action on $N$ (slight perturbations of $\zeta$ in the Lie algebra of $\operatorname{Isom}(N, g)$ will not introduce zeroes of the corresponding vector field on $N$ ). Hence $N$ must be a Seifert manifold.

Conversely, given a Seifert fibered manifold $N$, denote by $\zeta$ the generator of the fixed-point-free $S^{1}$ action and for each $\theta \in S^{1}$ denote by $\mathcal{R}_{\theta}$ its action on $N$. Define $\mathfrak{M}_{\zeta}$ as the collection of Riemann metrics $g$ on $N$ such that $|\zeta(x)|_{g} \equiv 1$. Note that $\mathfrak{M}$ is convex and

$$
\mathcal{R}_{\theta}^{*} \mathfrak{M}_{\zeta} \subset \mathfrak{M}_{\zeta}
$$

The $S^{1}$-average of any $g \in \mathfrak{M}_{\zeta}$ is $\zeta$-invariant and thus defines a Killing m.a.c structure on $N$. 


\subsection{The structural equations of a m.a.c. manifold.}

Consider an oriented m.a.c. 3 manifold $(N, \eta, g)$ and denote by $\nabla$ the Levi-Civita connection of the metric $g$. Fix an adapted local frame of $\left\{\zeta_{0}, \zeta_{1}, \zeta_{2}\right\}$ and denote by $\left\{\eta^{0}, \eta^{1}, \eta^{2}\right\}$ the dual coframe. The connection 1 -form of $\nabla$ with respect to these trivializations can be computed using Cartan's structural equation. More precisely, if

$$
d\left[\begin{array}{l}
\eta^{0} \\
\eta^{1} \\
\eta^{2}
\end{array}\right]=\left[\begin{array}{ccc}
0 & -A & B \\
A & 0 & -C \\
-B & C & 0
\end{array}\right] \wedge\left[\begin{array}{l}
\eta^{0} \\
\eta^{1} \\
\eta^{2}
\end{array}\right]
$$

$(A, B, C$ are real valued 1 -forms locally defined on $N$ ) then

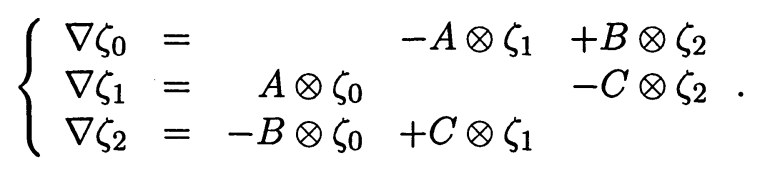

We will analyze the above equations when $(N, g)$ is a Killing m.a.c. manifold. For $j=0,1,2$ we set

$$
A_{j}=i_{\zeta_{j}} A, B_{j}=i_{\zeta_{j}} B, C_{j}=i_{\zeta_{j}} C .
$$

Since $L_{\zeta} g=0$ we have the equality

$$
g\left(\nabla_{X} \zeta, Y\right)=-g\left(X, \nabla_{Y} \zeta\right) \quad \forall X, Y \in \operatorname{Vect}(N) .
$$

We substitute $X$ and $Y$ with pairs of basic vectors $\zeta_{i}, \zeta_{j}$ and we obtain the identities

$$
A_{0}=B_{0}=A_{1}=B_{2}=0
$$

and

$$
A_{2}(x)=B_{1}(x) .
$$

Set $\lambda(x):=A_{2}(x)=B_{1}(x)$. The structural equations now yield

$$
d \eta=2 \lambda(x) * \eta .
$$

Thus the scalar $\lambda(x)$ is independent of the local frame used i.e. it is an invariant of the Killing m.a.c. structure $(N, \eta, g)$. 
Differentiating the above equality we deduce $0=2 d(\lambda(x) * \eta)$ so that

$$
\partial_{\zeta} \lambda(x)=0
$$

Note that

$$
\nabla_{\zeta} \zeta_{1}=-C_{0}(x) \zeta_{2}, \quad \nabla_{\zeta} \zeta_{2}=C_{0}(x) \zeta_{1} .
$$

Thus $C_{0}(x)$ defines the infinitesimal rotation of $\langle\zeta\rangle^{\perp}$ produced by the parallel transport along $\zeta$. Hence this is another invariant of the Killing m.a.c. structure and will be denoted by $\varphi(x)$. Finally set

$$
b(x)=\lambda(x)+\varphi(x) .
$$

Note for further references that

$$
\left[\zeta_{1}, \zeta_{0}\right]=\nabla_{\zeta_{1}} \zeta_{0}-\nabla_{0} \zeta_{1}=b(x) \zeta_{2}
$$

and

$$
\left[\zeta_{2}, \zeta_{0}\right]=\nabla_{\zeta_{2}} \zeta_{0}-\nabla_{\zeta_{0}} \zeta_{2}=-b(x) \zeta_{1} .
$$

We can now easily compute the sectional curvatures $\left\langle R\left(\zeta_{j}, \zeta\right) \zeta, \zeta_{j}\right\rangle$. More precisely, we have

$$
\begin{aligned}
R\left(\zeta_{1}, \zeta\right) \zeta & =\left(\nabla_{\zeta_{1}} \nabla_{\zeta}-\nabla_{\zeta} \nabla_{\zeta_{1}}-\nabla_{\left[\zeta_{1}, \zeta\right]}\right) \zeta \\
& =-\nabla_{\zeta}\left(\lambda(x) \zeta_{2}\right)-b(x) \nabla_{\zeta_{2}} \zeta=\lambda^{2}(x) \zeta_{1}
\end{aligned}
$$

Hence

$$
\left\langle R\left(\zeta_{1}, \zeta\right) \zeta, \zeta_{1}\right\rangle=\lambda^{2}(x)
$$

and similarly,

$$
\left\langle R\left(\zeta_{2}, \zeta\right) \zeta, \zeta_{2}\right\rangle=\lambda^{2}(x) .
$$

Hence the scalar curvature of $N$ is determined by

$$
s=2 \kappa+4 \lambda^{2}(x)
$$

where $\kappa(x)$ denotes the sectional curvature of the plane spanned by $\zeta_{1}$ and $\zeta_{2}$.

For each $\delta>0$ denote by $g_{\delta}$ the anisotropic deformation of $g$ defined by

$$
\begin{aligned}
g_{\delta}(X, X) & =g(X, X) \text { if } g(X, \zeta) \equiv 0 \\
g_{\delta}(\zeta, \zeta) & =\frac{1}{\delta^{2}} .
\end{aligned}
$$


Set

$$
\eta_{\delta}=\eta / \delta
$$

Note that $d \eta_{\delta}=2 \lambda \delta^{-1} *_{\delta} \eta_{\delta}$. Set $\lambda_{\delta}=\frac{\lambda}{\delta}$. Note that

$$
b_{\delta}=\delta b \quad \varphi_{\delta}=\delta b-\lambda / \delta=\delta \varphi+\left(\delta-\frac{1}{\delta}\right) \lambda .
$$

Anisotropic deformations as above were also discussed in [YK] where they were named D-homotheties.

The connection $\nabla$ defines via orthogonal projections a connection $\nabla^{\perp}$ on the complex line bundle Ann $\eta=\langle\zeta\rangle^{\perp}$. (This complex structure is defined by $\mathrm{i} \zeta_{1}=\zeta_{2}$.) More precisely

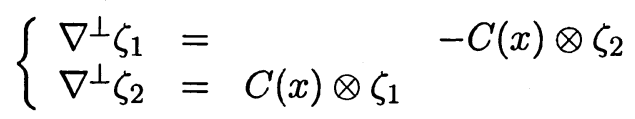

where $C(x)=\varphi(x) \eta+C_{1}(x) \eta_{1}+C_{2}(x) \eta_{2}$. Using the complex structure in $\langle\zeta\rangle^{\perp}$ we can locally describe $\nabla^{\perp}$ as

$$
\nabla^{\perp}=d-\mathrm{i} C(x)
$$

Under anisotropic deformations this connection 1-form changes to

$$
C_{\delta}=\varphi_{\delta} \eta_{\delta}+C_{1} \eta_{2}+C_{2} \eta_{2}=\frac{1}{\delta} \varphi_{\delta} \eta+C_{1} \eta_{2}+C_{2} \eta_{2}
$$

The equality (1.12) shows that as $\delta \rightarrow \infty$ the form $C_{\delta}$ converges to

$$
C_{\infty}:=b(x) \eta+C_{1}(x) \eta_{1}+C_{2}(x) \eta_{2}=\lambda(x) \eta+C(x) .
$$

If we denote by $\nabla^{\infty}$ the limiting connection we have

$$
\nabla^{\infty}=\nabla^{\perp}-\mathbf{i} \lambda(x) \eta
$$

Denote by $F^{\perp}$ the curvature of the connection $\nabla^{\perp}$ and by $\sigma$ the scalar

$$
\sigma=\left\langle F^{\perp}\left(\zeta_{1}, \zeta_{2}\right) \zeta_{2}, \zeta_{1}\right\rangle
$$

It is not difficult to show that $\sigma$ is independent of the local frame and so is an invariant of the Killing m.a.c. structure. With respect to this frame it has the description

$$
\sigma(x)=\partial_{\zeta_{1}} C_{2}-\partial_{\zeta_{2}} C_{1}-\left(C_{1}\right)^{2}-\left(C_{2}\right)^{2}
$$


Note that the anisotropic deformation introduced above does not change $\nabla^{\perp}$ so that

$$
\sigma_{\delta}(x)=\sigma(x) .
$$

Using the structural equations of $\nabla$ we deduce

$$
\begin{gathered}
\nabla_{\zeta_{1}} \nabla_{\zeta_{2}} \zeta_{2}=\left(\partial_{\zeta_{1}} C_{2}\right) \zeta_{1}-C_{1} C_{2} \zeta_{2} \\
\nabla_{\zeta_{2}} \nabla_{\zeta_{1}} \zeta_{2}=\left(\lambda C_{1}-\partial_{\zeta_{2}} \lambda\right) \zeta+\left(\lambda^{2}(x) \zeta_{1}+\partial_{\zeta_{2}} C_{1}\right) \zeta_{1}-C_{1} C_{2} \zeta_{2} \\
{\left[\zeta_{1}, \zeta_{2}\right]=-2 \lambda(x) \zeta+C_{1} \zeta_{1}+C_{2} \zeta_{2}}
\end{gathered}
$$

Hence

$$
\begin{aligned}
\kappa(x) & =\left\langle R\left(\zeta_{1}, \zeta_{2}\right) \zeta_{2}, \zeta_{1}\right\rangle \\
& =\partial_{\zeta_{1}} C_{2}-\partial_{\zeta_{2}} C_{1}-\left(C_{1}\right)^{2}-\left(C_{2}\right)^{2}-\lambda^{2}(x)+2 \lambda(x) \varphi(x) \\
& =\sigma(x)-\lambda^{2}(x)+2 \lambda(x) \varphi(x) .
\end{aligned}
$$

In particular, using (1.11) we deduce

$$
s(x)=2\left\{\sigma(x)+\lambda^{2}(x)+2 \lambda(x) \varphi(x)\right\} .
$$

Note that

$$
s_{\delta}(x)=2\left\{\sigma(x)+\lambda^{2} / \delta^{2}+2 \lambda(b-\lambda / \delta) / \delta\right\}
$$

so that

$$
\lim _{\delta \rightarrow \infty} s_{\delta}(x)=2 \sigma(x) .
$$

Thus for $\delta$ very large $\sigma(x)$ is a good approximation for the scalar curvature.

When the invariant $\lambda(x)$ is constant we will call the structure $(N, \eta, g)$ a $(K, \lambda)$-manifold. The $(K, 1)$-manifolds are also known as $K$-contact manifolds (cf. $[\mathrm{B}],[\mathrm{YK}]$ ). In dimension 3 this notion coincides with the notion of Sasakian manifold.

On a $(K, \lambda)$ manifold the sectional curvature of any plane containing $\zeta$ is $\lambda^{2}$ and the full curvature tensor is completely determined by the the sectional curvature $\kappa$ of the planes spanned by $\zeta_{1}$ and $\zeta_{2}$. The scalar curvature of $N$ is

$$
s=2 \kappa+4 \lambda^{2} .
$$

Using the structural equations we deduce that with respect to the adapted frame $\left\{\zeta_{0}, \zeta_{1}, \zeta_{2}\right\}$ the Ricci curvature has the form

$$
\operatorname{Ric}=\left[\begin{array}{ccc}
2 \lambda^{2} & 0 & 0 \\
0 & \kappa+\lambda^{2} & -\kappa \\
0 & -\kappa & \kappa+\lambda^{2}
\end{array}\right]
$$


For any oriented $(K, \lambda) 3$-manifold (not necessarily compact) we denote by $\mathfrak{K}_{N}$ the group of isomorphism of the $(K, \lambda)$ structure i.e. orientation preserving isometries which invariate $\eta$. For any discrete subgroup $\Gamma \subset \mathfrak{K}_{N}$ acting freely and discontinuously on $N$ we obtain a covering

$$
N \rightarrow N / \Gamma \text {. }
$$

Clearly $N / \Gamma$ admits a natural $(K, \lambda)$ structure induced from $N$. In $\S 1.4$ we will use this simple observation to construct $(K, \lambda)$-structures on any Seifert 3-manifold.

\subsection{The Boothby-Wang construction.}

In this subsection we describe some natural $(K, \lambda)$ structures on the total space of a principal $S^{1}$-bundle over a compact oriented surface. Except some minor modifications this construction is due to Boothby and Wang, [BW] (see also [B]).

Consider $\ell \in \mathbb{Z}$ and denote by $N_{\ell}$ the total space of a degree $\ell$ principal $S^{1}$ bundle over a compact oriented surface of genus $g: S^{1} \hookrightarrow N_{\ell} \stackrel{\pi}{\rightarrow} \Sigma$. We orient $N_{\ell}$ using the rule

$$
\operatorname{det} T N_{\ell}=\operatorname{det} T S^{1} \wedge \operatorname{det} T \Sigma .
$$

Assume $\Sigma$ is equipped with a Riemann metric $h_{b}$ such that $\operatorname{vol}_{h_{b}}(\Sigma)=\pi$. Recall that if $\omega \in \mathbf{i} \Omega^{1}\left(N_{\ell}\right)$ is a connection form on $N_{\ell}$ then $d \omega$ descends to a 2-form $\Omega$ on $\Sigma$, the curvature of $\omega$. Moreover

$$
\int_{\Sigma} c_{1}(\Omega)=\frac{\mathbf{i}}{2 \pi} \int_{\Sigma} \Omega=\ell=\int_{\Sigma} \frac{\ell}{\pi} d v_{h_{b}} .
$$

Notice in particular that $\mathrm{i} \Omega$ is cohomologous to $2 \ell d v_{h_{b}}$. Now pick a connection form $\omega$ such that

$$
\text { i } \Omega=2 \ell d v_{h_{b}} .
$$

Denote by $\zeta$ the unique vertical vector field on $N_{\ell}$ such that $\omega(\zeta) \cong \mathbf{i}$ and set $\eta_{\delta}=-\mathbf{i} \omega / \delta$. Thus $\eta_{\delta}(\zeta) \cong 1 / \delta$. Notice that Ann $\eta$ coincides with the horizontal distribution $H_{\omega}$ defined by the connection $\omega$. Now define a metric $h_{\delta}$ on $N_{\ell}$ according to the prescriptions.

$$
h_{\delta}(\zeta, \zeta)=1 / \delta^{2}
$$

and

$$
h_{\delta}(X, Y)=h_{b}\left(\pi_{*} X, \pi_{*} Y\right) \text { if } X, Y \text { are horizontal. }
$$


Clearly $L_{\zeta} h=$ i.e. $\zeta$ is a Killing vector field. Moreover

$$
\delta d \eta_{\delta}=-\Omega=-2 \ell \pi^{*} d v_{h_{b}}=-2 \ell * \eta_{\delta} .
$$

In other words, we have constructed a $(K,-\ell / \delta)$ structure on $N_{\ell}$.

We conclude this subsection by describing the geometry of $N_{\ell}$ in terms of the geometry of $\Sigma$. The only thing we need to determine is the curvature $\kappa$ introduced in the previous subsection in terms of the sectional curvature $\Sigma$. It is not difficult to see this coincides with the invariant $\sigma(x)$ defined in (1.14). Because of the special geometry of this situation formula (1.15) can be further simplified.

To achieve this we will use again the structural equations. Denote by $\left\{\psi^{1}, \psi^{2}\right\}$ a local, oriented orthonormal coframe on $\Sigma$ and set $\eta^{j}=\pi^{*} \psi^{j}$, $j=1,2$. Then $\left\{\eta^{0}=\eta, \eta^{1}, \eta^{2}\right\}$ is an adapted coframe on $N_{\ell}$.

The structural equations of $\Sigma$ have the form

$$
d\left[\begin{array}{l}
\psi^{1} \\
\psi^{2}
\end{array}\right]=\left[\begin{array}{cc}
0 & -\theta \\
\theta & 0
\end{array}\right] \wedge\left[\begin{array}{l}
\psi^{1} \\
\psi^{2}
\end{array}\right]
$$

where $\theta$ is a 1 -form locally defined on $\Sigma$. Set

$$
\theta_{j}=i_{\pi_{*} \zeta_{j}} \theta, \quad j=1,2
$$

Since $d \eta^{j}=\pi^{*} d \psi^{j}$ we deduce that $d \eta^{j}$ is horizontal. On the other hand, using (1.2) we deduce

$$
d \eta^{1}=A \wedge \eta^{0}-C \wedge \eta^{2}=\left(A_{2}+C_{0}\right) \eta^{2} \wedge \eta^{0}-C_{1} \eta^{1} \wedge \eta^{2} .
$$

This implies $C_{0}=-A_{2}=\ell / \delta$ so that

$$
b(x) \equiv 0
$$

and

$$
\varphi \equiv \ell / \delta
$$

Using (1.17) we deduce $d \psi^{1}=-\theta \wedge \psi^{2}=-\theta_{1} \psi^{1} \wedge \psi^{2}$ which yields

$$
C_{1}=\theta_{1} \text {. }
$$

Similarly one shows

$$
C_{2}=\theta_{2}
$$


Using the above two equalities and (1.13) we conclude that the limiting connection $\nabla^{\infty}$ on $\mathcal{K}$ is none-other than the pullback by $\pi: N \rightarrow \Sigma$ of the Levi-Civita connection on the canonical line bundle $K_{\Sigma} \rightarrow \Sigma$ i.e.

$$
\nabla^{\infty}=\pi^{*} \nabla^{K} .
$$

Using (1.15) and (1.19) we deduce

$$
\kappa=\sigma-3 \ell^{2} / \delta^{2}
$$

and in particular the scalar curvature of $N_{\ell}$ is given by

$$
s_{N}=2\left(\sigma-\ell^{2} / \delta^{2}\right) .
$$

Remark 1.4. Let $N$ denote the total space of a principal $S^{1}$ bundle over an oriented surface $\Sigma$, not necessarily compact. If $\omega$ denotes a connection form on $N$ such that -idw descends to a constant multiple of the volume form on $\Sigma$ then the previous computations extend verbatim to this case and one sees that in this situation one also obtains a $(K, \lambda)$-structure on $N$.

For example, let $N$ denote the unit tangent bundle of the hyperbolic plane $\mathbb{H}^{2}$. The Levi-Civita connection on $\mathbb{H}^{2}$ induces an $S^{1}$-connection $\omega$ on $N$. Then

$$
-\mathbf{i} d \omega=-1 d v o l_{\mathbb{H}^{2}}
$$

since $\mathbb{H}^{2}$ has constant curvature $\equiv-1$. Thus $N$ has a natural $(K, 1)(=$ Sasakian) structure.

The group of isometries of $\mathbb{H}^{2}$ is $P S L(2, \mathbb{R})$ and induces an action on $N$ which preserves the above Sasakian structure. (In fact, $N$ is isomorphic with $\operatorname{PSL}(2, \mathbb{R})$ and via this isomorphism the above action is precisely the usual left action of a Lie group on itself.) If now $\Gamma \subset P S L(2, \mathbb{R})$ is a Fuchsian group with a compact fundamental domain then $N / \Gamma$ is a compact Seifert manifold with a natural Sasakian structure.

\subsection{Geometric Seifert structures.}

The main result of this subsection shows that any compact, oriented, Seifert 3-manifold admits a $(K, \lambda)$ structure. This will follow easily from the description of the geometric Seifert structures in [JN] or [S].

Theorem 1.5. Any compact, oriented, Seifert 3-manifold admits a $(K, \lambda)$ structure. 
Proof. We will begin by reviewing the basic facts about the geometric Seifert structures in a form suitable to the application we have in mind. For details we refer to [JN] or [S] and the references therein.

A geometric structure on a manifold $M$ is a complete locally homogeneous Riemann metric of finite volume. The universal cover of a manifold $M$ equipped with a geometric structure is a homogeneous space which we will call the model of the structure. It is known that if a 3-manifold admits a geometric structure then its model belongs to a list of 8 homogeneous spaces (see $[\mathrm{S}]$ ).

Any Seifert manifold admits a geometric structure corresponding to one of the following 6 models:

$$
S^{2} \times \mathbb{E}^{1}, \quad \mathbb{E}^{3}, \quad \mathbb{H}^{2} \times \mathbb{E}^{1}, \quad S^{3}, \quad N, \quad P \tilde{S} L
$$

where $\mathbb{E}^{k}$ denotes the $k$-dimensional Euclidean space, $\mathbb{H}^{2}$ denotes the hyperbolic plane, $N$ denotes the Heisenberg group equipped with a left invariant metric and $P \tilde{S} L$ denotes the universal cover of $P S L(2, \mathbb{R}) \cong \operatorname{Isom}_{+}\left(\mathbb{H}^{2}\right)$.

According to [RV], the Seifert manifolds which admit $N$ as a model are the nontrivial $S^{1}$ bundles over a torus and, as we have seen in the previous subsection, such manifolds admit $(K, \lambda)$ structures.

The Seifert manifolds which admit geometric structures modeled by $\mathbb{E}^{3}$ are flat space form and are completely described in [Wo]. One can verify directly that these admit natural $(K, \lambda)$ structures.

$S^{3}$ has a natural $(K, 1)$ structure as the total space of the Hopf fibration $S^{3} \rightarrow S^{2}$. Any Seifert manifold modeled by $S^{3}$ is obtained as the quotient by a finite group of fiber preserving isometries. Thus they all inherit a $(K, 1)$ structure.

If $\mathbb{X}$ is a model other than $S^{3}$ or $\mathbb{E}^{3}$ then the group of isomorphisms which fix a given point $x \in \mathbb{X}$ fixes a tangent direction at that point. So $\mathbb{X}$ has an Aut $(\mathbb{X})$-invariant tangent line field. This line field fibers $\mathbb{X}$ over $\mathbb{S}^{2}$, $\mathbb{E}^{2}$ or $\mathbb{H}^{2}$.

For $\mathbb{X}=\mathbb{S}^{2} \times \mathbb{E}^{1}, \mathbb{H}^{2} \times \mathbb{E}^{1}$ this is the obvious fibration. $P \tilde{S} L$ can be alternatively identified with the universal cover of the unit tangent bundle $T_{1} \mathbb{H}^{2}$ of $\mathbb{H}^{2}$. It thus has a natural line fibration which coincides with the fibration abstractly described above. Note that the $(K, 1)$ structure on $T_{1} \mathbb{H}_{2}$ constructed at the end of $\S 1.3$ lifts to the universal cover $P \tilde{S} L$.

If $\mathbb{X}$ is one of of these remaining three models denote by $\operatorname{Aut}_{f}(X) \subset$ Aut $(X)$ the subgroup preserving the above line fibration (as an oriented fibration). Note that each of them admits a $(K, \lambda)$ structure and Aut $f_{f}$ is in fact a group of isomorphisms of this structure. 
The trivial Seifert manifold $S^{2} \times S^{1}$ presents a few "pathologies" as far as geometric Seifert structures are concerned (see [JN]) but we do not need to worry since it obviously admits a $(K, 0)$ structure.

The other Seifert manifolds which admit geometric structures modeled by $S^{2} \times \mathbb{E}^{1}, \mathbb{H}^{2} \times \mathbb{E}^{1}$ or $P \tilde{S} L$ can be obtained as quotients $\Pi \backslash \mathbb{X}$ where $\Pi$ is some subgroup of Aut $_{f}$. Thus $\Pi$ invariates the universal $(K, \lambda)$ structures on these models and therefore the quotients will admit such structures as well. The list of Seifert manifolds is complete.

Remark 1.6. The above analysis can be refined to offer an answer to the question raised in [We]: which Seifert manifolds admit Sasakian structures. The answer is simple. A Seifert manifold admits a Sasakian structure if and only if its (rational) Euler class is negative. According to [NR], these are precisely the Seifert manifolds which can occur as links of a quasi-homogeneous singularity. This extends (in the $3 D$ case) the previous result of [Sas] concerning Sasakian structures on Brieskorn manifolds.

This fact was observed by many other authors (see [ENS], [Ne], [V]). In fact, this geometry of the link is in most cases a complete invariant of the analytic type of the singularity (see [Ne], [Sch]).

\section{Dirac operators on 3-manifolds.}

In this section we discuss the relationships between $\operatorname{spin}^{c}$ structures and m.a.c. structures on a 3-manifold. On any m.a.c. 3-manifold, besides $\operatorname{spin}^{c}$ Dirac operators there exists another natural Dirac operator which imitates the Hodge-Dolbeault operator on a complex manifold. We will analyze the relationships between them.

\subsection{3-dimensional spinorial algebra.}

We include here a brief survey of the basic facts about the representations of $\operatorname{Spin}(3) \cong S U(2)$. . Denote by $\mathrm{Cl}_{3}$ the Clifford algebra generated by $V=\mathbb{R}^{3}$ and by $\mathbb{H}$ the skew-field of quaternions. Consider an orthogonal basis $\left\{e_{0}, e_{1}, e_{2}\right\}$ of $V$.

It is convenient to identify $\mathbb{H}$ with $\mathbb{C}^{2}$ via the correspondence

$$
\mathbb{H} \ni q=u+\mathbf{j} v \mapsto\left[\begin{array}{l}
u \\
v
\end{array}\right] \in \mathbb{C}^{2}
$$


where

$$
q=a+b \mathbf{i}+c \mathbf{j}+d \mathbf{k}, \quad u=a+b \mathbf{i}, \quad v=c-d \mathbf{i} .
$$

For each quaternion $q$ denote by $L_{q}$ (resp. $R_{q}$ ) the left (resp. the right) multiplication by $q$. $R_{\mathbf{i}}$ defines a complex structure on $\mathbb{H}$ and the correspondence (2.1) defines an isomorphism of complex vector spaces.

The Clifford algebra $C l_{3}$ can be represented on $\mathbb{C}^{2} \cong \mathbb{H}$ using the correspondences

$$
\begin{aligned}
& e_{0} \mapsto L_{\mathbf{i}} \longleftrightarrow \mathbf{c}\left(e_{0}\right)=\left[\begin{array}{cc}
\mathbf{i} & 0 \\
0 & -\mathbf{i}
\end{array}\right] . \\
& e_{1} \mapsto L_{\mathbf{j}} \longleftrightarrow \mathbf{c}\left(e_{1}\right)=\left[\begin{array}{cc}
0 & 1 \\
-1 & 0
\end{array}\right] . \\
& e_{2} \mapsto L_{\mathbf{k}} \longleftrightarrow \mathbf{c}\left(e_{2}\right)=\left[\begin{array}{ll}
0 & \mathbf{i} \\
\mathbf{i} & 0
\end{array}\right] .
\end{aligned}
$$

Note that $\operatorname{tr}\left(L_{\mathbf{i}} L_{\mathbf{j}} L_{\mathbf{k}}\right)=-2$ which shows that the above representation of $\mathrm{Cl}_{3}$ is the "positive" representation in the sense described in Lemma 1.22 of $[\mathrm{BC}]$. In [MOY] the "negative" representation is employed and thus the induced Dirac operators will differ by a sign from the Dirac operators in this paper.

The restriction of the above representation to $\operatorname{Spin}(3) \subset C l_{3}$ defines the complex spinor representation of $\operatorname{Spin}(3)$

$$
\text { c }: \operatorname{Spin}(3) \rightarrow \operatorname{Aut}\left(\mathbb{S}_{3}\right) .
$$

The Clifford multiplication map

$$
\text { c }: \Lambda^{*} V \stackrel{\mathfrak{q}}{\rightarrow} C l_{3} \rightarrow \text { End }\left(\mathbb{S}_{3}\right)
$$

identifies $\Lambda^{1} V$ with the space of traceless, skew-hermitian endomorphisms of $\mathbb{S}_{3}$. It extends by complex linearity to a map from $\Lambda^{1} V \otimes \mathbb{C}$ to the space of traceless endomorphisms of $\mathbb{S}_{3}$. In particular, the purely imaginary 1-forms are mapped to selfadjoint endomorphisms.

For each $\phi \in \mathbb{S}_{3}$ denote by $\tau(\phi)$ the endomorphism of $\mathbb{S}_{3}$ defined by $\tau(\phi)=\bar{\phi} \otimes \phi-\frac{1}{2}|\phi|^{2}$ i.e.

$$
\tau(\phi) \psi=\langle\psi, \phi\rangle \phi-\frac{1}{2}|\phi|^{2} \psi
$$


The map $\tau$ plays a central role in the 3-dimensional Seiberg-Witten equations.

If we identify $\mathbb{S}_{3}$ with $\mathbb{C}^{2}$ as above and if $\phi$ has the form

$$
\phi=\left[\begin{array}{l}
\alpha \\
\beta
\end{array}\right]
$$

then $\tau(\phi)$ has the description

$$
\tau(\phi)=\frac{1}{2} \sum_{i=0}^{2}\left\langle\phi, \mathbf{c}\left(e_{i}\right) \phi\right\rangle \mathbf{c}\left(e_{i}\right)=\left[\begin{array}{cc}
\frac{1}{2}\left(|\alpha|^{2}-|\beta|^{2}\right) & \alpha \bar{\beta} \\
\bar{\alpha} \beta & \frac{1}{2}\left(|\beta|^{2}-|\alpha|^{2}\right)
\end{array}\right] .
$$

It is not difficult to check that the nonlinear map

$$
\tau: \mathbb{S}_{3}: \rightarrow \operatorname{End}\left(\mathbb{S}_{3}\right)
$$

is $\operatorname{Spin}(3)$ equivariant.

We want to describe some of the invariant-theoretic features of the structure:

(oriented Euclidean 3 dimensional space + distinguished unit vector).

This is the algebraic counterpart of a m.a.c. structure on an oriented 3manifold.

Assume $V$ is an oriented Euclidean space which has a distinguished unit vector, say $e_{0}$. The group of isomorphisms of this structure is $U(1) \cong S^{1} \cong$ $S O(2)$. The group $\operatorname{Spin}(3)$ acts naturally on $V$. The Lie algebra of the subgroup $H$ of $\operatorname{Spin}(3)$ which fixes $e_{0}$ is generated by $e_{1} e_{2}=\mathfrak{q}\left(* e_{0}\right)$ and can be identified with $\underline{u}(1)$ via the correspondence

$$
e_{1} e_{2} \mapsto \mathbf{i} \in \underline{u}(1) .
$$

This tautologically identifies $H$ with $S^{1}$. The representation of $H$ on $\mathbb{S}_{3}$ is no longer irreducible and consequently $\mathbb{S}_{3}$ splits as a direct sum of irreducible $H$-modules. Alternatively, this splitting can be described as the unitary spectral decomposition of $\mathbb{S}_{3}$ defined by the action of $e_{1} e_{2}$ on $\mathbb{S}_{3}$. According to $(2.3)$ and (2.4) we have

$$
\mathbb{S}_{3} \cong \mathbb{S}_{3}(\mathbf{i}) \oplus \mathbb{S}_{3}(-\mathbf{i})
$$

The action of $H$ on $\mathbb{S}_{3}(\mathbf{i})$ is the tautological $S^{1}$ representation, while the action on $\mathbb{S}_{3}(-\mathbf{i})$ is the conjugate of the tautological representation. 


\subsection{3-dimensional spin geometry.}

Consider a compact, oriented, m.a.c. 3-manifold $(N, \eta, g)$. Since $w_{2}(N)=0$ the manifold $N$ is spin. To understand the relationship between the spin structures on $N$ and the m.a.c structure we need to consider gluing data of $T N$ compatible with the m.a.c. structure.

Consider a good, open cover $\left\{U_{\alpha}\right\}$ of $N$ and a gluing cocycle

$$
g_{\alpha \beta}: U_{\alpha \beta} \rightarrow S O(2) \cong U(1)
$$

defining $T N$. The cocycle is valued in $S O(2)$ since $T N$ has a distinguished section $\zeta$, the dual of $\eta$. Note that $g_{\alpha \beta}$ defines a complex structure in the real 2-plane bundle $\langle\zeta\rangle^{\perp}$. It is not difficult to see that $\langle\zeta\rangle^{\perp} \cong \mathcal{K}^{-1}$ as complex line bundles.

A spin structure on $T N$ is a lift of this cocycle to an $H$-valued cocycle, where $H$ is the subgroup of $\operatorname{Spin}(3)$ defined at the end of $\S 2.1$. Using the tautological identification $H \cong S^{1}$ we can identify the cover $H \rightarrow S O(2)$ with

$$
S^{1} \stackrel{z^{2}}{\rightarrow} S^{1}
$$

Hence a spin structure is defined by a cocycle

$$
\tilde{g}_{\alpha \beta}: U(1) \cong S O(2) \rightarrow U(1)
$$

such that $\tilde{g}_{\alpha \beta}^{2}=g_{\alpha \beta}$. In other words, $\tilde{g}_{\alpha \beta}$ defines a square root of $\mathcal{K}^{-1}$. Moreover, two such lifts define isomorphic square roots if and only if they are cohomologous so there exists a bijective correspondence between the square roots of $\mathcal{K}^{-1}$ (or, equivalently $\mathcal{K}$ ) and the spin structures on $N$.

Now, fix a spin structure on $N$ defined by a lift

$$
\tilde{g}_{\alpha \beta}: U_{\alpha \beta} \rightarrow H \text {. }
$$

The complex spinor bundle $\mathbb{S}$ of this spin structure is associated to the principal $H$-bundle defined by (2.7) via the representation

$$
H \hookrightarrow \operatorname{Spin}(3) \rightarrow \operatorname{Aut}(\mathbb{S}) .
$$

As we have already seen this splits as $\tau_{1} \oplus \tau_{-1}$ where $\tau_{1}$ denotes the tautological representation of $S^{1} \cong H$ and $\tau_{-1}$ is its conjugate.

The component $\tau_{1}$ defines the square root of of $\langle\zeta\rangle^{\perp}$ i.e. the line bundle $\mathcal{K}^{-1 / 2}$ characterizing the chosen spin structure. We have thus shown that a choice of a m.a.c. structure on $N$ produces a splitting

$$
\mathbb{S} \cong \mathcal{K}^{-1 / 2} \oplus \mathcal{K}^{1 / 2}
$$


Once we have fixed a spin structure it is very easy to classify the $\operatorname{spin}^{c}$ structures: they are bijectively parameterized by the complex line bundles $L \rightarrow N$. The complex spinor bundle associated to the $\operatorname{spin}^{c}$ structure defined by the line bundle $L$ is

$$
\mathbb{S}_{L} \cong \mathbb{S} \otimes L \cong \mathcal{K}^{-1 / 2} \otimes L \oplus \mathcal{K}^{1 / 2} \otimes L
$$

An important important special case is when $L=\mathcal{K}^{-1 / 2}$. In this case

$$
\mathbb{S}_{\mathcal{K}^{-1 / 2}} \cong \mathcal{K}^{-1} \oplus \underline{\mathbb{C}} \stackrel{\text { def }}{=} \mathbb{S}_{\eta}
$$

where we denoted by $\underline{\mathbb{C}}$ the trivial complex line bundle over $N$.

Remark 2.1. Our sign conventions differ from those of [MST]. There they chose $\mathbb{S}(\mathbf{i}) \cong \mathcal{K}^{1 / 2}$. The overall effect is a permutation of rows and columns in the block description of the geometric Dirac operator of $\S 2.4$.

\subsection{Pseudo Dolbeault operators.}

The complex bundle $\mathbb{S}_{\eta}$ introduced in $\S 2.2$ was a priori defined in terms of a fixed spin structure on $N$ but a posteriori, the spin structure becomes irrelevant. This is similar to complex manifolds where $\Lambda^{0, *} T^{*}$ is a complex spinor bundle of the $\operatorname{spin}^{c}$ structure canonically associated to the complex manifold. In that case the Dolbeault operator is a Dirac operator compatible with the Clifford structure. Moreover it is a geometric Dirac operator if the manifold is Kähler.

In this subsection want to pursue this analogy a little further. In the process we will construct an operator which behaves very much like the Dolbeault operator in the complex case.

Denote by $\mathcal{P}$ the real 2-plane bundle $\langle\eta\rangle^{\perp}$. We orient $\mathcal{P}$ using the complex structure $-\mathfrak{c}(* \eta)$ which identifies it with $\mathcal{K}$. Consider now a complex hermitian vector bundle $E \rightarrow N$. Any connection $\nabla$ on $E$ defines an operator

$$
\nabla: C^{\infty}(E) \rightarrow C^{\infty}\left(T^{*} N \otimes E\right)
$$

Now observe that

$$
T^{*} N \otimes E \cong(\langle\eta\rangle \oplus \mathcal{P}) \otimes E \cong E \oplus(\mathcal{K} \otimes E) \oplus\left(\mathcal{K}^{-1} \otimes E\right) .
$$

Hence for any section $\psi \in C^{\infty}(E)$ the covariant derivative $\psi$ orthogonally splits into three components:

$$
\nabla_{\zeta} \psi \in C^{\infty}(E)
$$




$$
{ }^{b} \nabla \psi \in C^{\infty}(\mathcal{K} \otimes E)
$$

and

$$
{ }^{b} \bar{\nabla} \psi \in C^{\infty}\left(\mathcal{K}^{-1} \otimes E\right) .
$$

It terms of a local adapted frame $\left\{\zeta_{0}=\zeta, \zeta_{1}, \zeta_{2}\right\}$ we have

$$
\begin{gathered}
{ }^{b} \nabla \psi=\varepsilon \otimes\left(\nabla_{1}-i \nabla_{2}\right) \psi \\
{ }^{b} \bar{\nabla} \psi=\bar{\varepsilon} \otimes\left(\nabla_{1}+i \nabla_{2}\right)
\end{gathered}
$$

where

$$
\nabla_{j}=\nabla_{\zeta_{j}}, \quad \varepsilon=\frac{1}{\sqrt{2}}\left(\eta^{1}+\mathbf{i} \eta^{2}\right), \quad \bar{\varepsilon}=\frac{1}{\sqrt{2}}\left(\eta^{1}-\mathbf{i} \eta^{2}\right) .
$$

For example when $E=\underline{\mathbb{C}}$ then ${ }^{b} \bar{\nabla} \psi \in C^{\infty}\left(\mathcal{K}^{-1}\right)$. In this case if $\nabla$ is the trivial connection $d$ we will write $\partial_{b}$ (resp. $\bar{\partial}_{b}$ ) instead of ${ }^{b} \nabla$ (resp. ${ }^{b} \bar{\nabla}$ ). Notice that if $E=\mathcal{K}^{-1}$ then ${ }^{b} \nabla \psi \in C^{\infty}(\underline{\mathbb{C}})$.

Coupling a metric connection $\nabla^{E}$ on $E$ with the Levi-Civita connection $\nabla^{\perp}$ on $\mathcal{K}^{ \pm}$we obtain connections $\nabla^{E, \pm}$ on $E \otimes \mathcal{K}^{ \pm}$and in particular operators

$$
{ }^{b} \nabla^{E,-}: C^{\infty}\left(E \otimes \mathcal{K}^{-}\right) \rightarrow C^{\infty}(E),{ }^{b} \bar{\nabla}^{E,+}: C^{\infty}(E \otimes \mathcal{K}) \rightarrow C^{\infty}(E) .
$$

Using the above explicit form of the operators ${ }^{b} \nabla$ and ${ }^{b} \bar{\nabla}$ and the structural equations of the background m.a.c structure we obtain the following result.

Lemma 2.2. For any hermitian vector bundle $E$ and any hermitian connection $\nabla^{E}$ on $E$ we have

$$
{ }^{b} \nabla^{*}={ }^{b} \bar{\nabla}^{E,+},{ }^{b} \bar{\nabla}^{*}={ }^{b} \nabla^{E,-}
$$

where the upper * denotes the formal adjoint.

The Levi-Civita connection on $(N, g)$ induces via orthogonal projection a connection $\nabla^{\perp}$ on $\mathcal{K}^{-1}$ compatible with the hermitian structure. The pseudo Dolbeault operator on $(N, \eta, g)$ is the first order partial differential operator $\mathfrak{d}: C^{\infty}\left(\mathbb{S}_{\eta}\right) \rightarrow C^{\infty}\left(\mathbb{S}_{\eta}\right)$ which in terms of the splitting $\mathbb{S}_{\eta} \cong \mathcal{K}^{-1} \otimes \underline{\mathbb{C}}$ has the block decomposition

$$
\mathfrak{d}_{N}=\left[\begin{array}{cc}
\mathbf{i} \nabla_{\zeta}^{\perp} & \bar{\partial}_{b} \\
{ }^{b} \nabla^{\perp} & -\mathbf{i} \partial_{\zeta}
\end{array}\right]
$$


where ${ }^{b} \nabla^{\perp}$ is obtained as above starting from the connection $\nabla^{\perp}$ on $\mathcal{K}^{-1}$. More generally, consider the complex spinor bundle $\mathbb{S}_{L}$ associated to the $\operatorname{spin}^{c}$ structure defined by the complex line bundle $L$

$$
\mathbb{S}_{L} \cong \mathcal{K}^{-1 / 2} \otimes L \oplus \mathcal{K}^{1 / 2} \otimes L .
$$

Using the connection $\nabla$ on $\mathcal{K}$ and a connection $A$ on $L$ we obtain connections $\nabla^{ \pm}$on $\mathcal{K}^{ \pm 1 / 2} \otimes L$. We can produce a twisted pseudo-Dolbeault operator $\mathfrak{d}_{L}=\mathfrak{d}_{L, A}$ on $\mathbb{S}_{L}$ described by the block decomposition

$$
\mathfrak{d}_{L}=\mathfrak{d}_{L, A}=\left[\begin{array}{cc}
\mathbf{i} \nabla_{\zeta}^{-} & { }^{b} \bar{\nabla}^{+} \\
{ }^{b} \nabla^{-} & -\mathbf{i} \nabla_{\zeta}^{+}
\end{array}\right] .
$$

Lemma 2.2 shows that $\mathfrak{d}_{L}$ is formally selfadjoint. Note that

$$
\mathfrak{d}_{N}=\mathfrak{d}_{\mathcal{K}^{-1 / 2}}
$$

When $L$ is trivial we set $\mathfrak{d}_{L}=\mathfrak{d}_{0}$.

Denote by $\mathbf{F}^{ \pm}$the curvature of $\nabla^{ \pm}$. We want to analyze the "commutator"

$$
\left.{ }^{b} \bar{\nabla}^{+}, \nabla_{\zeta}^{+}\right] \stackrel{\text { def }}{=}{ }^{b} \bar{\nabla}^{+} \circ \nabla_{\zeta}^{+}-\nabla_{\zeta}^{-} \circ{ }^{b} \bar{\nabla}^{+} \text {. }
$$

assuming $N$ is a Killing m.a.c manifold. Choose a local adapted frame $\left\{\zeta, \zeta_{1}, \zeta_{2}\right\}$ with dual coframe $\left\{\eta, \eta_{1}, \eta_{2}\right\}$. For any $\psi \in C^{\infty}\left(\mathcal{K}^{1 / 2} \otimes L\right)$ we have

$$
\begin{aligned}
{\left[{ }^{b} \bar{\nabla}^{+}, \nabla_{\zeta}^{+}\right] \psi=} & \bar{\varepsilon} \otimes\left(\nabla_{1}^{+}+\mathbf{i} \nabla_{2}^{+}\right) \nabla_{\zeta}^{+} \psi-\nabla_{\zeta}^{-}\left\{\bar{\varepsilon} \otimes\left(\nabla_{1}^{+}+\mathbf{i} \nabla_{2}^{+}\right)\right\} \psi \\
= & \bar{\varepsilon} \otimes\left(\nabla_{1}^{+}+\mathbf{i} \nabla_{2}^{+}\right) \nabla_{\zeta}^{+} \psi-\left(\nabla^{+} \bar{\varepsilon}\right) \otimes\left(\nabla_{1}^{+}+\mathbf{i} \nabla_{2}^{+}\right) \psi \\
& -\bar{\varepsilon} \otimes\left(\nabla_{\zeta}^{+}\left(\nabla_{1}^{+}+\mathbf{i} \nabla_{2}^{+}\right)\right) \psi \\
= & \bar{\varepsilon} \otimes\left\{\left(\nabla_{1}^{+}+\mathbf{i} \nabla_{2}^{+}\right) \nabla_{\zeta}^{+}-\nabla_{\zeta}^{+}\left(\nabla_{1}^{+}+\mathbf{i} \nabla_{2}^{+}\right)\right\} \psi \\
& -\left(\nabla_{\zeta}^{+} \bar{\varepsilon}\right) \otimes\left(\nabla_{1}^{+}+\nabla_{2}^{+}\right) \psi
\end{aligned}
$$

(use $\left.\nabla_{\zeta}^{\frac{1}{\zeta}} \bar{\varepsilon}=-\mathbf{i} \varphi(x) \bar{\varepsilon}\right)$

$$
\begin{aligned}
= & \bar{\varepsilon} \otimes\left(\mathbf{F}^{+}\left(\zeta_{1}, \zeta\right)+\mathbf{i F}^{+}\left(\zeta_{2}, \zeta\right)+\nabla_{\left[\zeta_{1}, \zeta\right]}^{+}+\mathbf{i} \nabla_{\left[\zeta_{2}, \zeta\right]}^{+}\right) \psi \\
& +\mathbf{i} \varphi(x) \bar{\varepsilon} \otimes\left(\nabla_{1}^{+}+\mathbf{i} \nabla_{2}^{+}\right) \psi
\end{aligned}
$$

(use (1.7) and (1.8))

$$
=\bar{\varepsilon} \otimes\left(\mathbf{F}^{+}\left(\zeta_{1}, \zeta\right)+\mathbf{i F}^{+}\left(\zeta_{2}, \zeta\right)+b(x) \nabla_{2}^{+}-\mathbf{i} b(x) \nabla_{1}^{+}\right) \psi
$$




$$
\begin{aligned}
& +\mathbf{i} \varphi(x) \bar{\varepsilon} \otimes\left(\nabla_{1}^{+}+\mathbf{i} \nabla_{2}^{+}\right) \psi \\
= & \bar{\varepsilon} \otimes\left\{\mathbf{F}^{+}\left(\zeta_{1}, \zeta\right)+\mathbf{i F ^ { + }}\left(\zeta_{2}, \zeta\right)-\mathbf{i} b(x)\left(\nabla_{1}^{+}+\mathbf{i} \nabla_{2}^{+}\right)\right\} \psi \\
& +\mathbf{i} \varphi(x) \bar{\varepsilon} \otimes\left(\nabla_{1}^{+}+\mathbf{i} \nabla_{2}^{+}\right) \psi \\
= & \bar{\varepsilon} \otimes\left(\mathbf{F}^{+}\left(\zeta_{1}, \zeta\right)+\mathbf{i F}^{+}\left(\zeta_{2}, \zeta\right)\right) \psi-\mathbf{i} \lambda \bar{\varepsilon} \otimes\left(\nabla_{1}^{+}+\mathbf{i} \nabla_{2}^{+}\right) \psi
\end{aligned}
$$

We have thus proved

$$
\left.{ }^{b} \bar{\nabla}^{+}, \nabla_{\zeta}^{+}\right]=-\mathbf{i} \lambda(x)^{b} \bar{\nabla}^{+}+\bar{\varepsilon} \otimes\left\{\mathbf{F}^{+}\left(\zeta_{1}, \zeta\right)+\mathbf{i F ^ { + }}\left(\zeta_{2}, \zeta\right)\right\}
$$

When dealing with the Seiberg-Witten equations it is convenient to describe the curvature term in the above formula in terms of the curvature $F_{A}$ of $L$. We will use the formula

$$
\mathbf{F}^{+}=F_{\mathcal{K}^{1 / 2} \otimes L}=F_{A}+\frac{1}{2} F_{\mathcal{K}} .
$$

Hence we need to explicitly describe the curvature of $\mathcal{K}$ equipped with the connection $\nabla^{\perp}$.

Note that $\mathcal{K}^{-1}$ can be identified with the bundle $\langle\zeta\rangle^{\perp}$ equipped with the complex structure

$$
\mathbf{i} \zeta_{1}=\zeta_{2} \quad \mathbf{i} \zeta_{2}=-\zeta_{1}
$$

We will compute the curvature of this line bundle using the structural equations of $\nabla^{\perp}$

$$
\nabla^{\perp} \zeta_{j}=-\mathbf{i} C \otimes \zeta_{j} j=1,2 .
$$

Assuming $N$ is a Killing m.a.c manifold we deduce after some simple manipulations

$$
\begin{aligned}
& F_{\mathcal{K}^{-1}}\left(\zeta_{1}, \zeta\right)=-\mathbf{i}\left(-\partial_{1} \varphi(x)+\partial_{\zeta} C_{1}+b(x) C_{2}\right) \\
& F_{\mathcal{K}^{-1}}\left(\zeta_{2}, \zeta\right)=-\mathbf{i}\left(-\partial_{2} \varphi(x)+\partial_{\zeta} C_{2}-b(x) C_{1}\right)
\end{aligned}
$$

Set $\mu(x)=C_{1}+\mathbf{i} C_{2}$. Some elementary algebra shows

$$
F_{\mathcal{K}^{-1}}\left(\zeta_{1}, \zeta\right)+\mathbf{i} F_{\mathcal{K}^{-1}}\left(\zeta_{2}, \zeta\right)=\mathbf{i}\left(\partial_{1}+\mathbf{i} \partial_{2}\right) \varphi-\left(\mathbf{i} \partial_{\zeta}+b(x)\right) \mu
$$

Hence

$$
\bar{\varepsilon} \otimes\left(F_{\mathcal{K}}\left(\zeta_{1}, \zeta\right)+\mathbf{i} F_{\mathcal{K}}\left(\zeta_{2}, \zeta\right)=-\mathbf{i} \bar{\partial}_{b} \varphi+\bar{\varepsilon} \otimes\left\{\left(\mathbf{i} \partial_{\zeta}+b(x)\right) \mu\right\} .\right.
$$

We now want to clarify the "mysterious" term $\left(\mathbf{i} \partial_{\zeta}+b(x)\right) \mu$ in the above formula. To achieve this we will use the structural equations (1.2). Thus

$$
d \eta^{1}=A \wedge \eta-C \wedge \eta^{2}=-b(x) \eta \wedge \eta^{2}-C_{1} * \eta
$$


and

$$
d \eta^{2}=-B \wedge \eta+C \wedge \eta^{1}=b(x) \eta \wedge \eta^{1}-C_{2} * \eta .
$$

Temporarily set $\omega=\sqrt{2} \varepsilon=\eta^{1}+\mathbf{i} \eta^{2}$. The above equalities yield

$$
d \omega=\mathbf{i} b(x) \eta \wedge \omega-\mu * \eta .
$$

Differentiating the last equality we deduce

$$
d(\mu * \eta)=\mathrm{i} d(b(x) \eta \wedge \omega)
$$

i.e.

$$
\left(\partial_{\zeta} \mu\right) d v o l_{g}=\mathbf{i}\{d b(x) \wedge \eta \wedge \omega+b(x) d \eta \wedge \omega-b(x) \eta \wedge d \omega\} .
$$

The middle term in the right-hand-side of the above formula cancels. The third term can be computed using (2.11). Hence

$$
\left(\partial_{\zeta} \mu\right) d v o l_{g}=\mathbf{i}\{d b(x) \wedge \eta \wedge \omega+b(x) \eta \wedge * \eta\}
$$

or equivalently,

$$
\left(\partial_{\zeta}-\mathbf{i} b(x)\right) \mu d v o l_{g}=\mathbf{i} d b(x) \wedge \eta \wedge \omega .
$$

Since

$$
d b(x) \wedge \eta \wedge \omega=\left(-\mathbf{i} \partial_{1} b(x)+\partial_{2} b(x)\right) d v o l_{g}
$$

we deduce

$$
\left(\mathbf{i} \partial_{\zeta}+b(x)\right) \mu=\mathbf{i}\left(\partial_{1}+\mathbf{i} \partial_{2}\right) b(x) .
$$

In a more invariant form

$$
\bar{\varepsilon} \otimes\left(\mathbf{i} \partial_{\zeta}+b(x)\right) \mu=\mathbf{i} \bar{\partial}_{b} b(x) .
$$

Using the above equality in (2.10) we deduce

$$
\bar{\varepsilon} \otimes\left(F_{\mathcal{K}}\left(\zeta_{1}, \zeta\right)+\mathbf{i} F_{\mathcal{K}}\left(\zeta_{2}, \zeta\right)\right\}=-\mathbf{i} \bar{\partial}_{b} \varphi+\mathbf{i} \bar{\partial}_{b} b(x)=\mathbf{i} \bar{\partial}_{b} \lambda(x)
$$

Set

$$
F_{A}^{0,1}:=\bar{\varepsilon} \otimes F_{A}\left(\zeta, \zeta_{1}+\mathbf{i} \zeta_{2}\right)
$$

and

$$
F_{A}^{1,0}:=\varepsilon \otimes F_{A}\left(\zeta, \zeta_{1}-\mathbf{i} \zeta_{2}\right)
$$

We can now rephrase the commutativity relation (2.9) as

$$
\left[{ }^{b} \bar{\nabla}^{+}, \nabla_{\zeta}^{+}\right]=-\mathbf{i} \lambda(x)^{b} \bar{\nabla}^{+}+\frac{\mathbf{i}}{2} \bar{\partial}_{b} \lambda(x)-F_{A}^{0,1}
$$


By passing to formal adjoints we deduce using Lemma 2.2

$$
\begin{aligned}
{\left[{ }^{b} \nabla^{-}, \nabla_{\zeta}^{-}\right]^{*} } & =\left[\left({ }^{b} \bar{\nabla}^{+}\right)^{*}, \nabla_{\zeta}^{-}\right]:=\left({ }^{b} \bar{\nabla}^{+}\right)^{*} \nabla_{\zeta}^{-}-\nabla_{\zeta}^{+}\left({ }^{b} \bar{\nabla}^{+}\right)^{*} \\
& =\mathbf{i} \lambda\left({ }^{b} \bar{\nabla}^{+}\right)^{*}-\frac{\mathbf{i}}{2}\left({ }^{b} \bar{\partial}\right)^{*} \lambda+F_{A}^{1,0} .
\end{aligned}
$$

On a $(K, \lambda)$ manifold the commutativity relations (2.14) and (2.14) further simplify. Fix a $\operatorname{spin}^{c}$ structure determined by a line bundle $L$ and choose a connection $A$ on $L$. It will be extremely convenient to introduce two new differential operators $Z_{A}, T_{A}: C^{\infty}\left(\mathbb{S}_{L}\right) \rightarrow C^{\infty}\left(\mathbb{S}_{L}\right)$ defined by the block decompositions

$$
\begin{aligned}
Z_{A} & =\left[\begin{array}{cc}
\mathbf{i} \nabla_{\zeta}^{A,-} & 0 \\
0 & -\mathbf{i} \nabla_{\zeta}^{A,+}
\end{array}\right] \\
T_{A} & =\left[\begin{array}{cc}
0 & b \bar{\nabla}^{+} \\
\left({ }^{b} \bar{\nabla}^{+}\right)^{*} & 0
\end{array}\right] .
\end{aligned}
$$

Then the commutativity relations (2.14) and (2.15) can be simultaneously rephrased as an anti-commutator identity

$$
\left\{Z_{A}, T_{A}\right\}:=Z_{A} T_{A}+T_{A} Z_{A}=-\lambda T_{A}+\mathbf{i}\left[\begin{array}{cc}
0 & F_{A}^{0,1} \\
-F_{A}^{1,0} & 0
\end{array}\right]
$$

We want to point out that the operators $Z_{A}, T_{A}$ and $F_{A}^{0,1}$ depend on the background m.a.c. structure. Both $Z_{A}$ and $F_{A}^{0,1}$ will be affected by anisotropic deformations while $T_{A}$ is invariant. We will write $Z_{A, \delta}$ and $F_{A, \delta}^{0,1}$ to emphasize this dependence. Clearly

$$
F_{A, \delta}^{0,1}=\delta F_{A}^{0,1} .
$$

To describe the dependence $\delta \mapsto Z_{A, \delta}$ we will use the equality (1.13) of $\S 1.2$ describing the dependence on $\delta$ of the Levi-Civita connection on $\mathcal{K}^{-1}$. More precisely define

$$
Z_{A, \infty}:=\left[\begin{array}{cc}
\mathbf{i} \hat{\nabla}_{\zeta}^{A,-} & 0 \\
0 & -\mathbf{i} \hat{\nabla}_{\zeta}^{A,+}
\end{array}\right]
$$

where for each connection $A$ on $L$ we denoted by $\hat{\nabla}^{A, \pm}$ the connection on $\mathcal{K}^{ \pm 1 / 2} \otimes L$ obtained by tensoring the limiting connection $\nabla^{\infty}$ on $\mathcal{K}^{ \pm 1 / 2}$ (described in (1.13)) with the connection $A$. We have

$$
\delta Z_{A, \infty}=Z_{A, \delta}+\frac{\lambda}{2 \delta} .
$$


Using this equality in (2.16) we deduce

$$
\left\{Z_{A, \infty}, T_{A}\right\}=\frac{\mathbf{i}}{\delta}\left[\begin{array}{cc}
0 & F_{A, \delta}^{0,1} \\
F_{A, \delta}^{1,0} & 0
\end{array}\right]=\mathbf{i}\left[\begin{array}{cc}
0 & F_{A}^{0,1} \\
-F_{A}^{1,0} & 0
\end{array}\right]
$$

\subsection{Geometric Dirac operators.}

In this subsection we will analyze the geometric Dirac operators on 3manifolds and in particular, we will relate them with the pseudo-Dolbeault operators of $\S 2.3$.

Consider an oriented Killing m.a.c manifold $(N, \eta, g)$ with a fixed spin structure. Denote by $\mathbb{S} \cong \mathcal{K}^{-1 / 2} \oplus \mathcal{K}^{1 / 2}$ the bundle of complex spinors associated to this structure.

We begin by first recalling the construction of the canonical connection on $\mathcal{K}$. Pick a local adapted frame $\left\{\zeta_{0}=\zeta, \zeta_{1}, \zeta_{2}\right\}$ and denote by $\sigma_{j}$ the Clifford multiplication by $\zeta_{j}, j=0,1,2$. With respect to the canonical decomposition $\mathbb{S} \cong \mathcal{K}^{-1 / 2} \oplus \mathcal{K}^{1 / 2}$ these operators have the descriptions

$$
\begin{aligned}
\sigma_{0} & =\left[\begin{array}{ll}
\mathbf{i} & 0 \\
0 & -\mathbf{i}
\end{array}\right] \\
\sigma_{1} & =\left[\begin{array}{ll}
0 & \bar{\varepsilon} \\
-\varepsilon & 0
\end{array}\right] \\
\sigma_{2} & =\left[\begin{array}{cc}
0 & \mathbf{i} \bar{\varepsilon} \\
\mathbf{i} \varepsilon & 0
\end{array}\right]
\end{aligned}
$$

where $\varepsilon$ (resp. $\bar{\varepsilon}$ ) denotes the tensor multiplication by $\bar{\varepsilon}$ (resp $\varepsilon$ )

$$
\bar{\varepsilon}: \mathcal{K}^{1 / 2} \rightarrow \mathcal{K}^{-1 / 2} \quad\left(\text { resp. } \varepsilon: \mathcal{K}^{-1 / 2} \rightarrow \mathcal{K}^{1 / 2}\right) .
$$

If $\left(\omega_{i j}\right)$ denotes the $\underline{s o}(3)$ valued 1-form associated to the Levi-Civita connection via the local frame $\left\{\zeta_{j}\right\}$ i.e.

$$
\nabla \zeta_{j}=\sum_{i} \omega_{i j} \zeta_{i}
$$

then the canonical connection on $\mathbb{S}$ is defined by

$$
\tilde{\nabla}=d-\frac{1}{2} \sum_{i<j} \omega_{i j} \otimes \sigma_{i} \sigma_{j}
$$


Using the structural equations (1.3) we deduce that, with respect to the local frame $\left\{\zeta_{j}\right\}$, the canonical connection on $\mathbb{S}$ has the form

$$
\tilde{\nabla}=d-\frac{1}{2}\left(A \otimes \sigma_{2}+B \otimes \sigma_{1}+C \otimes \sigma_{0}\right) .
$$

Using the fact that $N$ is a Killing m.a.c. we deduce

$$
\begin{gathered}
\tilde{\nabla}_{\zeta}=\partial_{\zeta}-\frac{1}{2}\left[\begin{array}{rl}
\mathbf{i} \varphi(x) & 0 \\
0 & -\mathbf{i} \varphi(x)
\end{array}\right] \\
\tilde{\nabla}_{1}=\partial_{\zeta_{1}}-\frac{1}{2}\left[\begin{array}{rr}
\mathbf{i} C_{1} & \lambda \bar{\varepsilon} \\
-\lambda \varepsilon & -\mathbf{i} C_{1}
\end{array}\right] \\
\tilde{\nabla}_{2}=\partial_{\zeta_{2}}-\frac{1}{2}\left[\begin{array}{rr}
\mathbf{i} C_{2} & \mathbf{i} \lambda \bar{\varepsilon} \\
\mathbf{i} \lambda \varepsilon & -\mathbf{i} C_{2}
\end{array}\right] .
\end{gathered}
$$

The canonical, untwisted (geometric) Dirac operator on $\mathbb{S}$ is defined by

$$
\begin{aligned}
\mathfrak{D}_{0} & =\mathfrak{D}_{\mathbb{S}}=\sigma_{0} \tilde{\nabla}_{0}+\sigma_{1} \tilde{\nabla}_{1}+\sigma_{2} \tilde{\nabla}_{2} \\
& =\left[\begin{array}{cc}
\mathbf{i}\left(\partial_{\zeta}-\mathbf{i} \varphi / 2\right) & \bar{\varepsilon} \otimes\left\{\left(\partial_{1}+\mathbf{i} C_{1} / 2\right)\right. \\
\varepsilon \otimes\left\{-\left(\partial_{1}+\mathbf{i} C_{1} / 2\right)\right. & \left.+\mathbf{i}\left(\partial_{2}+\mathbf{i} C_{2} / 2\right)\right\} \\
\left.+\mathbf{i}\left(\partial_{2}+\mathbf{i} C_{2} / 2\right)\right\} & -\mathbf{i}\left(\partial_{\zeta}+\mathbf{i} \varphi / 2\right)
\end{array}\right]+\lambda \mathbf{1}_{\mathbb{S}} .
\end{aligned}
$$

In terms of the pseudo-Dolbeault operator we have

$$
\mathfrak{D}_{\mathbb{S}}=\mathfrak{d}_{\mathbb{S}}+\lambda .
$$

More generally if we twist $\mathbb{S}$ by a line bundle $L$ equipped with a hermitian connection $A$ we obtain a geometric Dirac operator on $\mathbb{S}_{L}=\mathbb{S} \otimes L$ and as above one establishes the following identity

$$
\mathfrak{D}_{L}=\mathfrak{d}_{A}+\lambda=Z_{A}+T_{A}+\lambda .
$$

Clearly $\mathfrak{D}_{A}$ changes under the adiabatic deformations of the metric. More precisely, we deduce from the equality (2.17) that

$$
\mathfrak{D}_{A, \delta}=\delta Z_{A, \infty}+T_{A}+\frac{\lambda}{2 \delta}
$$

\section{The Seiberg-Witten equations.}

In this section we finally take-up the study of the 3-dimensional SeibergWitten equations. We will restrict our considerations to the special case 
when the 3-manifold $N$ has a $(K, \lambda)$-structure. When $\lambda=0$ it was observed by many authors that these equations can be solved quite explicitly. The situation is more complicated when $\lambda \neq 0$ for the reasons explained in the introduction. We subject $N$ to an anisotropic adiabatic deformation so that in the limit $\lambda_{\delta} \rightarrow 0$ and study the behavior of the solutions of the SeibergWitten equations as the metric degenerates. The solutions converge either to pairs (flat connections, zero spinor) or to vortices on the base of the Seifert manifold.

\subsection{Generalities.}

The goal of this subsection is to describe the 3-dimensional SeibergWitten equations and then derive a few elementary consequences.

Consider a compact, oriented m.a.c 3-manifold $(N, g)$. Fix a spin structure on $N$ defined by the square root $\mathcal{K}^{-1 / 2}$. The data entering the SeibergWitten equations are the following.

(a) $\mathrm{A} \operatorname{spin}^{c}$ structure determined by the line bundle $L$.

(b) A connection $A$ of $L \rightarrow N$.

(c) A spinor $\phi$ i.e. a section of the complex spinor bundle $\mathbb{S}_{L}$ associated to the given $\operatorname{spin}^{c}$ structure.

The connection $A$ defines a geometric Dirac operator $\mathfrak{D}_{A}$ on $\mathbb{S}_{L}$. The Seiberg-Witten equations are

$$
\left\{\begin{array}{rlr}
\mathfrak{D}_{A} \phi & = & 0 \\
\mathbf{c}\left(* F_{A}\right) & = & \tau(\phi)
\end{array}\right.
$$

where $*$ is the Hodge $*$-operator of the metric $g, \tau$ is defined in (2.6) and $\mathbf{c}$ is the Clifford multiplication described in (2.5). We will omit the symbol $\mathbf{c}$ when no confusion is possible.

The Seiberg-Witten equations have a variational nature. Fix a smooth connection $A_{0}$ on $L$ and define

$$
\mathfrak{f}: L^{1,2}\left(\mathbb{S}_{L} \oplus \mathbf{i} T^{*} N\right) \rightarrow \mathbb{R}
$$

by

$$
\mathfrak{f}(\psi, a)=\frac{1}{2} \int_{N} a \wedge\left(F_{A_{0}}+F_{A_{0}+a}\right)+\frac{1}{2} \int_{N}\left\langle\psi, \mathfrak{D}_{A_{0}+a} \psi\right\rangle d v_{g}
$$


Lemma 3.1. The differential of $\mathfrak{f}$ at a point $\mathfrak{c}=(\phi, a)$ is

$$
d_{\mathfrak{c}} \mathfrak{f}(\dot{\phi}, \dot{a})=\int\left\langle\dot{a}, \mathbf{c}^{-1}(\tau(\phi))-* F_{A_{0}+a}\right\rangle d v_{g}+\int_{N} \mathfrak{R e}\left\langle\dot{\phi}, \mathfrak{D}_{A_{0}+a} \phi\right\rangle d v_{g}
$$

Proof. Set $A=A_{0}+a$. We have

$$
\begin{aligned}
d_{\mathfrak{c}} \mathfrak{f}(\dot{\phi}, \dot{a})= & \left.\frac{d}{d t}\right|_{t=0} \mathfrak{f}(\phi+t \dot{\phi}, a+t \dot{a}) \\
= & \frac{1}{2} \int_{N} \dot{a} \wedge\left(F_{A_{0}}+F_{A}\right)+\frac{1}{2} \int_{N} a \wedge d \dot{a}-\int_{N} \mathfrak{R e}\left\langle\dot{\phi}, \mathfrak{D}_{A} \phi\right\rangle d v_{g} \\
& -\frac{1}{2} \int_{N}\langle\phi, \mathbf{c}(\dot{a}) \phi\rangle d v_{g}
\end{aligned}
$$

(Stokes)

$$
\begin{aligned}
= & \frac{1}{2} \int_{N} \dot{a} \wedge\left(F_{A_{0}}+F_{A}\right)+\frac{1}{2} \int_{N} \dot{a} \wedge d a \\
& -\frac{1}{2} \int_{N}\langle\phi, \mathbf{c}(\dot{a}) \phi\rangle d v_{g}-\int_{N} \mathfrak{R e}\left\langle\dot{\phi}, \mathfrak{D}_{A} \phi\right\rangle d v_{g} \\
= & \int_{N} \dot{a} \wedge F_{A}+\frac{1}{2} \int_{N}\langle\phi, \mathbf{c}(\dot{a}) \phi\rangle d v_{g}+\int_{N} \mathfrak{R e}\left\langle\dot{\phi}, \mathfrak{D}_{A} \phi\right\rangle d v_{g} .
\end{aligned}
$$

Since both $\dot{a}$ and $F_{A}$ are purely imaginary we get

$$
\int_{N}\left\langle\dot{a}, * F_{A}\right\rangle d v_{g}=-\int_{N} \dot{a} \wedge F_{A}
$$

where $*$ is the complex linear Hodge $*$-operator. On the other hand, a simple computation shows that $\left(\dot{a}=\sum_{i} \dot{a}_{i} \eta_{i}\right)$

$$
\begin{aligned}
\int_{N}\langle\phi, \mathbf{c}(\dot{a}) \phi\rangle d v_{g} & =-\int_{N} \sum_{i} \dot{a}_{i}\left\langle\phi, \mathbf{c}\left(\eta_{i}\right) \phi\right\rangle d v_{g} \\
& =-\int_{N} \sum_{i}\left\langle\dot{a}_{i} \eta_{i}, \overline{\left\langle\phi, \mathbf{c}\left(\eta_{i}\right) \phi\right\rangle} \eta_{i}\right\rangle d v_{g} \\
& =2 \int_{N}\left\langle\dot{a}, \mathbf{c}^{-1}(\tau(\phi))\right\rangle d v_{g} .
\end{aligned}
$$

Putting all the above together we get the lemma. 
The gauge group $\mathfrak{G}_{L}=\operatorname{Aut}(L) \cong \operatorname{Map}\left(N, S^{1}\right)$ acts on the space of pairs $(\psi, a)$ by

$$
\gamma \cdot(\psi, a):=\left(\gamma \cdot \psi, a-\gamma^{-1} d \gamma\right)
$$

Moreover

$$
\mathfrak{f}(\gamma \cdot(\psi, a))-\mathfrak{f}(\psi, a)=-\int_{N} \gamma^{-1} d \gamma \wedge F_{A_{0}}=2 \pi \mathbf{i} \int_{N} \gamma^{-1} d \gamma \wedge c_{1}\left(A_{0}\right)
$$

Thus $\mathfrak{f}$ is unchanged by the gauge transformations homotopic to the constants. We see that the critical points of $f$ are precisely the solutions of the Seiberg-Witten equations. In particular, the above considerations show that the moduli space of solutions is invariant under the action of $\mathfrak{G}_{L}$ and so it suffices to look at the quotient of this action.

The Seiberg-Witten equations have a more explicit description once we choose an adapted orthonormal frame $\zeta, \zeta_{1}, \zeta_{2}$. Using the decomposition $\mathbb{S}_{L} \cong \mathcal{K}^{-1 / 2} \otimes L \oplus \mathcal{K}^{1 / 2} \otimes L$ we can represent $\phi$ as

$$
\phi=\left[\begin{array}{c}
\alpha \\
\beta
\end{array}\right]
$$

If $N$ is a $(K, \lambda)$ - manifold and we denote by $\nabla^{ \pm}$the covariant derivatives induced by $A$ on $\mathcal{K}^{ \pm 1 / 2} \otimes L$ then the Seiberg-Witten equations can be rephrased as

$$
\left\{\begin{aligned}
+\lambda \nabla_{\zeta}^{-} \alpha+{ }^{b} \bar{\nabla}^{+} \beta & =0 \\
\left({ }^{b} \bar{\nabla}^{+}\right)^{*} \alpha-\mathbf{i} \nabla_{\zeta}^{+} \beta & =0 \\
\frac{1}{2}\left(|\alpha|^{2}-|\beta|^{2}\right) & =\mathbf{i} F_{A}\left(\zeta_{1}, \zeta_{2}\right) \\
\mathbf{i} \alpha \bar{\beta} & =\bar{\varepsilon} \otimes F_{A}\left(\zeta_{1}+\mathbf{i} \zeta_{2}, \zeta\right)=-F_{A}^{0,1}
\end{aligned}\right.
$$

In particular, note that

$$
\eta \wedge c_{1}(A)=\frac{\mathbf{i}}{2 \pi} F_{A}\left(\zeta_{1}, \zeta_{2}\right) \eta \wedge \eta^{1} \wedge \eta^{2}=\frac{1}{4 \pi}\left(|\alpha|^{2}-|\beta|^{2}\right) d v o l_{g} .
$$

Hence

$$
\int_{N} \eta \wedge c_{1}(A)=\frac{1}{4 \pi}\left(\|\alpha\|^{2}-\|\beta\|^{2}\right)
$$


where $\|\cdot\|$ denotes the $L^{2}$ - norm over $N$.

In terms of the operators $Z_{A}$ and $T_{A}$ defined in $\S 2.3$ we can rewrite the first two equations as

$$
\left(Z_{A}+T_{A}\right) \phi=-\lambda \phi .
$$

The anti-commutation relation (2.16) shows that if $(\phi, A)$ is a solution of the Seiberg-Witten equation then

$$
\lambda^{2}\left[\begin{array}{l}
\alpha \\
\beta
\end{array}\right]=\left(Z_{A}+T_{A}\right)^{2} \phi=Z_{A}^{2} \phi+T_{A}^{2} \phi-\lambda T_{A} \phi+\left[\begin{array}{cc}
0 & \alpha \bar{\beta} \\
\bar{\alpha} \beta & 0
\end{array}\right] \cdot\left[\begin{array}{c}
\alpha \\
\beta
\end{array}\right] .
$$

\subsection{Adiabatic limits.}

We now have all the data we need to study the behavior of the SeibergWitten equations as the metric is anisotropically deformed until it degenerates.

Let $(N, \eta, g)$ and $\mathcal{K}^{1 / 2}$ as above. As usual we denote by $\left(N, \eta_{\delta}, g_{\delta}\right)$ the anisotropic deformation defined in $\S 1.1$. For each $\delta \geq 1$ we will refer to the Seiberg-Witten equations defined in terms of the metric $g_{\delta}$ as the $S W_{\delta}$ equations. More explicitly these are $\left(|\zeta|_{g_{1}} \equiv 1,|\eta|_{g_{1}} \equiv 1\right)$

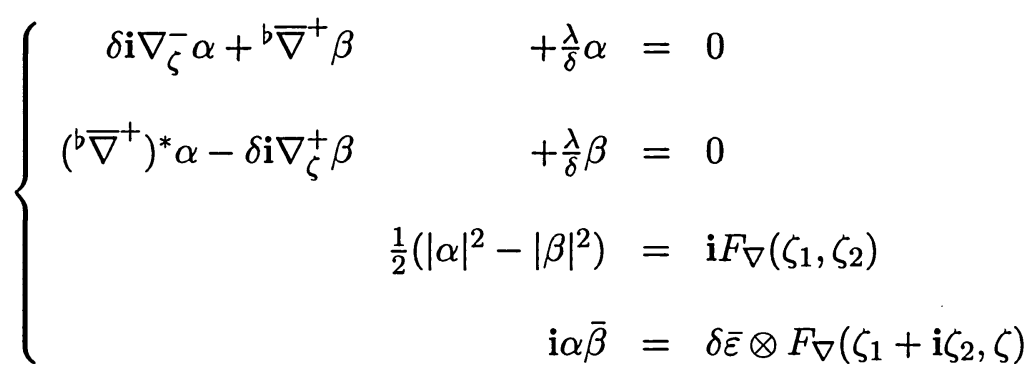

The operator $Z_{A}$ depends on the metric $g_{\delta}$ through the Levi-Civita connection $\nabla^{\perp}$ on $\mathcal{K}$ while $T_{A}$ is unaffected by the adiabatic changes in the metric. Note that

$$
\lim _{\delta \rightarrow \infty} \frac{1}{\delta} Z_{A, \delta}=Z_{A, \infty}
$$

We will fix a smooth connection $\nabla^{0}$ on $L$. The Sobolev norms will be defined in terms of this connection and its tensor products with the connections induced by the Levi-Civita connection of the fixed metric $g=g_{1}$. An arbitrary connection on $L$ will have the form

$$
\nabla^{0}+A_{\delta}, \quad A_{\delta} \in \underline{u}(1) \otimes \Omega^{1}(N) .
$$


For $1 \leq p \leq \infty$ we denoted by $\|\cdot\|_{p}$ the $L^{p}$-norm with respect to the metric $g=g_{1}$, by $\|\cdot\|$ the $L^{2}$-norm with respect to the same metric while $\|\cdot\|_{\delta}$ will denote the $L^{2}$-norm with respect to the metric $g_{\delta}$.

We denote by $\mathfrak{A}_{L}$ the affine space of smooth connections on $L$ and by $\mathcal{S}_{\delta}$ the collection of gauge equivalence classes of solutions of $S W_{\delta}$.

Theorem 3.2. Let $(N, \eta, g)$ as above and fix a $\operatorname{spin}^{c}$ structure defined by a complex line bundle $L$ (so that the associated complex spinor bundle has determinant $L^{2}$ ). Assume that for each sufficiently large $\delta$

$$
\mathcal{S}_{\delta} \neq \emptyset \text {. }
$$

Then any sequence $\left\{\left[\phi_{\delta}, A_{\delta}\right] \in \mathcal{S}_{\delta} ; \delta \gg 1\right\}$ admits a subsequence which converges in the $L^{1,2}$ topology to a pair

$$
[\phi, A] \in\left(\mathfrak{A}_{L} \times C^{\infty}(L)\right) / \operatorname{Aut}(L)
$$

satisfying the following conditions.

$$
\begin{gathered}
F_{A}(\zeta, \cdot) \equiv 0 \\
{ }^{b} \bar{\nabla}^{+} \beta=\hat{\nabla}_{\zeta}^{A,-} \alpha=0 \\
\left({ }^{b} \bar{\nabla}^{+}\right)^{*} \alpha=\hat{\nabla}_{\zeta}^{A,+} \beta=0 \\
\|\alpha\| \cdot\|\beta\|=0 .
\end{gathered}
$$

The equations (3.6) and (3.7) can be equivalently rephrased as

$$
Z_{A, \infty} \phi=T_{A} \phi=0 .
$$

Definition 3.3. A pair $(\phi, A)$ satisfying (3.5)-(3.8) will be called an adiabatic solution of the Seiberg-Witten equations. The collection of gauge equivalence classes of adiabatic solutions will be called the adiabatic moduli space corresponding to the fixed $\operatorname{spin}^{c}$ structure.

Proof. The proof of the theorem relies essentially on the following uniform estimates. 
Lemma 3.4. There exist $R_{1}, R_{2}>0$ such that

$$
\sup \left|\phi_{\delta}(x)\right| \leq R_{1} \quad \forall \delta \geq 1
$$

and

$$
\begin{gathered}
\left\|F\left(A_{\delta}\right)\right\|_{\infty} \leq R_{2} \quad \forall \delta \geq 1 . \\
\left\|\left|\alpha_{\delta}\right| \cdot\left|\beta_{\delta}\right|\right\|_{2}=O(1 / \delta) \quad \text { as } \delta \rightarrow \infty .
\end{gathered}
$$

Proof of the lemma. As in Lemma 2 of $[\mathrm{KM}]$ we deduce that

$$
\sup \left|\phi_{\delta}(x)\right| \leq \sup \left|s_{\delta}(x)\right|
$$

where $s_{\delta}$ denotes the scalar curvature of $g_{\delta}$. The estimate (3.10) is now a consequence of (1.16) in $\$ 1.2$.

To prove (3.11) note first that $F\left(A_{\delta}\right)$ splits into two orthogonal parts. A horizontal part

$$
F^{h}\left(A_{\delta}\right)=F_{12}\left(A_{\delta}\right) \eta^{1} \wedge \eta^{2}
$$

and a vertical part

$$
F^{v}\left(A_{\delta}\right)=F_{01}\left(A_{\delta}\right) \eta \wedge \eta^{1}+F_{20}\left(A_{\delta}\right) \eta^{2} \wedge \eta .
$$

The third equation in (3.4) coupled with (3.10) yields

$$
\left\|F^{h}\left(A_{\delta}\right)\right\|_{\infty}=O(1) \text { as } \delta \rightarrow \infty .
$$

The fourth equality in (3.4) implies

$$
\left\|F^{v}\left(A_{\delta}\right)\right\|_{\infty} \leq \text { const. } \delta^{-1}\left\|\left|\alpha_{\delta}\right| \cdot\left|\beta_{\delta}\right|\right\|_{\infty}=O\left(\delta^{-1}\right)
$$

where

$$
\phi_{\delta}=\left[\begin{array}{c}
\alpha_{\delta} \\
\beta_{\delta}
\end{array}\right] .
$$

To prove (3.12) we will use (3.3) and we get (writing $Z_{A_{\delta}}$ instead of the more accurate $Z_{A_{\delta}, \delta}$ )

$$
\begin{aligned}
\lambda_{\delta}^{2} \phi_{\delta} & =\left(Z_{A_{\delta}}+T_{A_{\delta}}\right)^{2} \phi_{\delta} \\
& =Z_{A_{\delta}}^{2} \phi_{\delta}+T_{A_{\delta}}^{2} \phi_{\delta}-\lambda_{\delta} T_{A_{\delta}} \phi_{\delta}+\left[\begin{array}{cc}
0 & \alpha_{\delta} \bar{\beta}_{\delta} \\
\overline{\alpha_{\delta}} \beta_{\delta} & 0
\end{array}\right]\left[\begin{array}{c}
\alpha_{\delta} \\
\beta_{\delta}
\end{array}\right] .
\end{aligned}
$$


Taking the inner product with $\phi_{\delta}$ in the above equality and then integrating by parts with respect to the metric $g_{\delta}$ we deduce

$$
\left\|Z_{A_{\delta}} \phi_{\delta}\right\|_{\delta}^{2}+\left\|T_{A_{\delta}} \phi_{\delta}\right\|_{\delta}^{2}+2\left\|\left|\alpha_{\delta}\right| \cdot\left|\beta_{\delta}\right|\right\|_{\delta}^{2}=\lambda_{\delta}^{2}\left\|\phi_{\delta}\right\|_{\delta}^{2}+\lambda_{\delta}\left\langle T_{A_{\delta}} \phi_{\delta}, \phi_{\delta}\right\rangle_{\delta} .
$$

The Cauchy inequality yields

$$
\begin{aligned}
\left\|Z_{A_{\delta}} \phi_{\delta}\right\|_{\delta}^{2}+\left\|T_{A_{\delta}} \phi_{\delta}\right\|_{\delta}^{2}+2\left\|\left|\alpha_{\delta}\right| \cdot\left|\beta_{\delta}\right|\right\|_{\delta}^{2} & \leq\left|\lambda_{\delta}\right| \cdot\left\|\phi_{\delta}\right\|_{\delta}\left(\left|\lambda_{\delta}\right| \cdot\left\|\phi_{\delta}\right\|_{\delta}+\left\|T_{A_{\delta}} \phi_{\delta}\right\|_{\delta}\right)
\end{aligned}
$$

Since $\left|\lambda_{\delta}\right|,\left\|\phi_{\delta}\right\|_{\delta}^{2}=O\left(\delta^{-1}\right)$ we first deduce from the above inequality that $\left\|T_{A_{\delta}} \phi_{\delta}\right\|_{\delta}=O\left(\delta^{-3 / 2}\right)$ and then

$$
\left\|\left|\alpha_{\delta}\right| \cdot\left|\beta_{\delta}\right|\right\|_{\delta}=O\left(\delta^{-3 / 2}\right) .
$$

Up to a rescaling this is precisely the inequality (3.12).

From the estimate (3.11) we deduce that $A_{\delta}$ (modulo Coulomb gauges) is bounded in the $L^{1, p}$-norm for any $1<p<\infty$. Thus a subsequence converges strongly in $L^{p}$ and weakly in $L^{1, p}$ to some Hölder continuous connection $A$ on $L$. The fourth equation in (3.4) and the estimate (3.12) we have just proved show that

$$
\left\|F_{A_{\delta}}^{0,1}\right\|=O\left(\delta^{-2}\right) .
$$

For each connection $A$ on $L$ set

$$
\mathbf{D}_{A, \delta}=\delta Z_{A, \infty}+T_{A}
$$

For $\delta=1$ we will write simply $\mathbf{D}_{A}$. Recall that $\mathbf{D}_{A, \delta}=\mathfrak{D}_{A, \delta}-\lambda_{\delta} / 2$.

The condition (3.9) is a consequence of the following auxiliary result.

Lemma 3.5 (Adiabatic decoupling lemma). Consider a sequence of smooth connections $A_{\delta} \in \mathfrak{A}_{L}$ satisfying the following conditions.

(i) $A_{\delta}$ converges in the weak $L^{1, p}$ topology $(p>3=\operatorname{dim} N)$ to a Hölder continuous connection $A$.

(ii) $\left\|F_{A_{\delta}}^{0,1}\right\|=o\left(\delta^{-1}\right)$ as $\delta \rightarrow \infty$.

Then any sequence $\phi_{\delta} \in C^{\infty}\left(\mathbb{S}_{L}\right)$ such that

$$
\left\|\phi_{\delta}\right\|=O(1) \text { as } \delta \rightarrow \infty
$$


and

$$
\left\|\mathfrak{D}_{A_{\delta}} \phi_{\delta}\right\|=o(1)
$$

contains a subsequence which converges strongly in $L^{1,2}\left(\mathbb{S}_{L}\right)$ to a spinor $\phi \epsilon$ $L^{1,2}\left(\mathbb{S}_{L}\right)$ satisfying

$$
Z_{A, \infty} \phi=T_{A} \phi=0 .
$$

(Above, for simplicity, we denoted by $\mathfrak{D}_{A_{\delta}}$ the Dirac operator $\mathfrak{D}_{A_{\delta}, \delta}$.)

Proof. Set $Z_{A_{\delta}}=Z_{A_{\delta}, \delta}, w_{\delta}=\mathfrak{D}_{A_{\delta}} \phi_{\delta}$ and $\Xi_{\delta}=\frac{1}{\delta} Z_{A_{\delta}}$. The coefficients of $\Xi_{\delta}$ converge uniformly to the coefficients of $Z_{A, \infty}$. More precisely, the condition (i) implies $\Xi_{\delta}=Z_{A, \infty}+R$ where the zeroth order term $R$ satisfies

$$
\|R\|_{\infty}=o(1)
$$

Note that although $\Xi_{\delta}$ is defined using the metric $g_{\delta}$ it is formally self-adjoint with respect to the metric $g_{1}$ as well.

The equation $\mathfrak{D}_{A_{\delta}} \phi_{\delta}=w_{\delta}$ can be rewritten as

$$
\left(Z_{A_{\delta}}+T_{A_{\delta}}\right) \phi_{\delta}=-\lambda_{\delta} \phi_{\delta}+w_{\delta}
$$

so that

$$
\left\|\left(Z_{A_{\delta}}+T_{A_{\delta}}\right) \phi_{\delta}\right\|^{2} \leq 2 \lambda_{\delta}^{2}\left\|\phi_{\delta}^{2}\right\|+o(1)
$$

Using the equation (2.16) we get

$$
\begin{aligned}
\left\|\left(Z_{A_{\delta}}+T_{A_{\delta}}\right) \phi_{\delta}\right\|^{2}= & \int_{N}\left\langle\left(Z_{A_{\delta}}+T_{A_{\delta}}\right)^{2} \phi_{\delta}, \phi_{\delta}\right\rangle d v_{g} \\
= & \left\|Z_{A_{\delta}} \phi_{\delta}\right\|^{2}+\left\|T_{A_{\delta}} \phi_{\delta}\right\|^{2}-\lambda_{\delta} \int_{N}\left\langle T_{A_{\delta}} \phi_{\delta}, \phi_{\delta}\right\rangle d v_{g} \\
& +\int_{N}\left\langle\mathcal{F}_{\delta} \phi_{\delta}, \phi_{\delta}\right\rangle d v_{g}
\end{aligned}
$$

where

$$
\mathcal{F}_{\delta}=\mathbf{i} \delta\left[\begin{array}{cc}
0 & F_{A_{\delta}}^{0,1} \\
F_{A_{\delta}}^{1,0} & 0
\end{array}\right]
$$

Hence

$$
\delta^{2}\left\|\Xi_{\delta} \phi_{\delta}^{2}\right\|^{2}+\left\|T_{A_{\delta}} \phi_{\delta}\right\|^{2} \leq\left\|\phi_{\delta}\right\|\left\{\left|\lambda_{\delta}\right| \cdot\left\|T_{A_{\delta}} \phi_{\delta}\right\|+\left\|\mathcal{F}_{\delta}\right\|+2 \lambda_{\delta}^{2}\left\|\phi_{\delta}\right\|+o(1)\right\} .
$$


Since $\left\|\phi_{\delta}\right\|=O(1)$ and $\left\|\mathcal{F}_{\delta}\right\|=O\left(\delta\left\|F_{A_{\delta}}^{0,1}\right\|\right)=o(1)$ we deduce

$$
\delta^{2}\left\|\Xi_{\delta} \phi_{\delta}^{2}\right\|^{2}+\left\|T_{A_{\delta}} \phi_{\delta}\right\|^{2} \leq C\left(\delta^{-1}\left\|T_{A_{\delta}} \phi_{\delta}\right\|+\delta^{-2}+o(1)\right) .
$$

This yields

$$
\left\|T_{A_{\delta}} \phi_{\delta}\right\|=o(1) \text { and }\left\|\Xi_{\delta} \phi_{\delta}\right\|=o\left(\delta^{-1}\right) .
$$

We know have

$$
\mathrm{D}_{A} \phi_{\delta}=\psi_{\delta}:=\Xi_{\delta} \phi_{\delta}+T_{A_{\delta}} \phi_{\delta}-R \phi_{\delta}
$$

The estimates (3.16) and (3.17) show that $\left\|\psi_{\delta}\right\|=o(1)$. The elliptic estimates applied to the fixed elliptic operator $\mathbf{D}_{A}$ (with Hölder continuous zeroth order part) show that

$$
\left\|\phi_{\delta}\right\|_{1,2} \leq C\left(\left\|\phi_{\delta}\right\|+\|\psi\|_{\delta}\right)
$$

Thus $\phi_{\delta}$ is bounded in the $L^{1,2}$-norm so a subsequence (still denoted by $\phi_{\delta}$ ) will converge strongly in $L^{2}$. Using again the elliptic estimates we get

$$
\left\|\phi_{\delta_{1}}-\phi_{\delta_{2}}\right\|_{1,2} \leq C\left(\left\|\phi_{\delta_{1}}-\phi_{\delta_{2}}\right\|+\left\|\psi_{\delta_{1}}\right\|+\left\|\psi_{\delta_{2}}\right\|\right) .
$$

This shows the (sub)sequence $\phi_{\delta}$ is Cauchy in the $L^{1,2}$ norm and in particular it must converge in this norm to a $\phi$ which must satisfy

$$
Z_{A, \infty} \phi=T_{A} \phi=0
$$

due to (3.17). The proof is complete.

We can now conclude the proof of the theorem. From the above lemma we know that $\phi_{\delta}$ converges in $L^{1,2}$. By Sobolev inequality the sequence $\phi_{\delta}$ must also converge in $L^{p}, 1 \leq p \leq 6$. The last two equations in (3.4) allow us to conclude that $F_{A_{\delta}}$ is actually convergent in $L^{3}$ so we can conclude that modulo Coulomb gauges the connections $A_{\delta}$ converge strongly in $L^{1,3}$.

Note that $\phi$ satisfies a condition slightly weaker than (3.8) namely

$$
\||\alpha| \cdot|\beta|\|=0 .
$$

We will now prove this implies (3.8). We can rewrite the limiting connection $\hat{\nabla}^{A}$ as a sum $\hat{\nabla}^{A}=\nabla^{0}+B$, where $B \in L^{1, p}\left(\operatorname{End}\left(\mathbb{S}_{L}\right)\right)$ for any $1 \leq p<\infty$. Note that $\alpha$ and $\beta$ satisfy the elliptic Dirac equation with Hölder continuous coefficients

$$
\mathfrak{D}_{\infty} \phi=0
$$


This equation can be rewritten as

$$
\mathfrak{D}_{\nabla}^{0} \phi+Q_{B} \phi=0
$$

where $Q_{B} \in L^{1, p}\left(\right.$ End $\left.\left(\mathbb{S}_{L}\right)\right), 1 \leq p<\infty$. Since $\phi \in L^{1,2}\left(\mathbb{S}_{L}\right) \cap L^{\infty}\left(\mathbb{S}_{L}\right)$ we deduce

$$
Q_{B} \phi \in L^{1,2}\left(\mathbb{S}_{L}\right) \text {. }
$$

Using this in (3.19) we deduce $\phi \in L^{2,2}$. Note that $\hat{\nabla}_{\zeta}^{ \pm}=\nabla_{\zeta}^{A, \pm} \pm \lambda / 2$ where $\nabla^{A}$ denotes the spinor connection on $\mathbb{S}_{L}$ obtained by tensoring the connection $A$ with the Levi-Civita connection defined by the metric $g_{1}$. Applying ${ }^{b} \bar{\nabla}^{+}$to the second equation in (3.18) and using the commutator equality (2.14) we deduce after some simple manipulations that

$$
{ }^{b} \bar{\nabla}^{b} \bar{\nabla}^{*} \alpha-\nabla_{\zeta}^{2} \alpha+|\beta|^{2} \alpha+\lambda^{b} \bar{\nabla} \beta=0 .
$$

Since ${ }^{b} \bar{\nabla} \beta=0$ and $\alpha \otimes \beta \equiv 0$ we deduce

$$
{ }^{b} \bar{\nabla}^{b} \bar{\nabla}^{*} \alpha-\nabla_{\zeta}^{2} \alpha=0
$$

We can rewrite the above equation as

$$
{ }^{b} \bar{\nabla}^{0}\left({ }^{b} \bar{\nabla}^{0}\right)^{*} \alpha-\nabla_{\zeta}^{2} \alpha+P(\nabla \alpha)+Q \alpha=0
$$

where the "coefficient" $P \in L^{1,2} \subset L^{6}$ while $Q \in L^{2}$ is at least $L^{2}$ (it is a linear combination of the connection $A$ and its derivatives) while the differential operator above is a generalized Laplacian. This is more than sufficient to apply the unique continuation principle in Thm. $4.3 \mathrm{of}[\mathrm{H}]$ to deduce that if $\alpha$ vanishes on an open subset of $N$ then it must vanish everywhere.

A dual argument proves a similar result for $\beta$. Since the product $\alpha \otimes \beta$ is identically zero we deduce that one of them must vanish on an open set and hence everywhere. The equality (3.8) is proved and this completes the proof of the theorem.

For later applications to Seiberg-Witten-Floer theory it is very important to present a more compact description of the adiabatic Seiberg-Witten equations (3.5)-(3.8). This is achieved in our next result.

Proposition 3.6. The following conditions are equivalent.

(i) The pair $(\psi, A)$ is an adiabatic solution of the Seiberg-Witten equations. 
(ii) The pair $(\psi, A)$ is a solutions of the equations

$$
\left(S W_{\infty}\right):\left\{\begin{aligned}
\mathfrak{D}_{A} \psi & =\frac{\lambda}{2} \psi \\
\mathbf{c}\left(* F_{A}\right) & =\tau(\psi)
\end{aligned}\right.
$$

Proof. Note that the first equation can be rephrased as $\mathbf{D}_{A} \psi=0$. The implication (i) $\Rightarrow$ (ii) is now obvious. We prove the converse. Set as usual $\psi=\alpha \oplus \beta$ The first equation of $\left(S W_{\infty}\right)$ implies

$$
0=\mathbf{D}_{A}^{2} \psi=Z_{A, \infty}^{2} \psi+T_{A}^{2} \psi+\left\{Z_{A, \infty}, T_{A}\right\} \psi
$$

Using (2.18) we deduce

$$
\left(Z_{A, \infty}^{2}+T_{A}^{2}+\mathbf{i}\left[\begin{array}{cc}
0 & F_{A}^{0,1} \\
-F_{A}^{1,0} & 0
\end{array}\right]\right)\left[\begin{array}{l}
\alpha \\
\beta
\end{array}\right]=0 .
$$

Taking the inner product with $\psi$ and then integrating by parts over $N$ (using the second equation of $\left.\left(S W_{\infty}\right)\right)$ we deduce

$$
\left\|Z_{A, \psi}\right\|^{2}+\left\|T_{A} \psi\right\|^{2}+2\||\alpha| \cdot|\beta|\|^{2}=0 .
$$

This last equality implies immediately the conditions (3.6) -(3.8) so that $(\psi, A)$ is indeed an adiabatic solution.

Remark 3.7. The equation $\left(S W_{\infty}\right)$ is also equivalent with $\left(S W_{\infty}\right)_{\delta}$ which is defined as $\left(S W_{\infty}\right)$ but using the deformed metric $g_{\delta}$ and its associated geometric objects instead of $g$.

\subsection{The Seiberg-Witten equations on circle bundles.}

When $N$ is the total space of a circle bundle over a surface the above result can be given a more precise form.

Let $N_{\ell}$ be the total space of a degree $\ell$ principal $S^{1}$ bundle

$$
S^{1} \hookrightarrow N_{\ell} \stackrel{\pi}{\rightarrow} \Sigma
$$

where $\Sigma$ is a compact surface of genus $g \geq 1$. Fix a complex structure on $\Sigma$ and denote by $K$ the canonical line bundle. Then

$$
\mathcal{K} \cong \pi^{*} K .
$$


Moreover, according to (1.22), in this case the limiting connection $\nabla^{\infty}$ on $\mathcal{K}$ coincides with the connection pulled back from $K$.

Now fix a spin structure on $\Sigma$ by choosing a square root $K_{\Sigma}^{1 / 2}$ on $\Sigma$. This defines by pullback a spin structure on $N$. Denote by $\left(N_{\ell}, \eta_{\delta}, h_{\delta}\right)$ the Boothby-Wang $(K, \lambda)$ structure described in $\S 1.3$.

Fix a $\operatorname{spin}^{c}$ structure on $N$ given by a line bundle $L_{N}$. If the SeibergWitten equations $S W_{\delta}$ corresponding to the above $\operatorname{spin}^{c}$ structure have solutions for every $\delta \gg 1$ we deduce from the above theorem that $L_{N}$ admits a connection $A$ such that $F_{A}(\zeta, \cdot) \equiv 0$. This implies $\pi_{*} F_{A} \equiv 0$ where $\pi_{*}$ denotes the integration along the fibers of $\pi: N_{\ell} \rightarrow \Sigma$. From the Gysin sequence of this fibration we deduce that $c_{1}\left(L_{N}\right) \in \pi^{*} H^{2}(\Sigma)$ i.e. $L_{N}$ is the pullback of a line bundle $L_{\Sigma} \rightarrow \Sigma$. In particular we have the following vanishing result.

Corollary 3.8. Fix a $\operatorname{spin}^{c}$ structure on $N_{\ell}$ which is not the pullback of any $\operatorname{spin}^{c}$ structure on $\Sigma$. Then the Seiberg-Witten equations $S W_{\delta}$ have no solutions for any $\delta \gg 1$. In particular, when $|\ell|=1$ then for any nontrivial $\operatorname{spin}^{c}$ structure on $N$ and for all $\delta \gg 1$ the equations $S W_{\delta}$ have no solutions.

Now fix a $\operatorname{spin}^{c}$ structure on $N_{\ell}$ defined by a complex line bundle $L_{N}=$ $\pi^{*} L_{\Sigma}$. Any adiabatic solution of Seiberg-Witten equation is a pair $(\phi, A)$ satisfying the following conditions.

(a) A connection $A$ on $L_{N}$ with horizontal curvature i.e. $F_{A}(\zeta, \cdot) \equiv 0$.

(b) Sections $\alpha \in \mathcal{K}^{-1 / 2} \otimes L_{N}$ and $\beta \in \mathcal{K}^{1 / 2} \otimes L_{N}$ such that

$$
\begin{gathered}
Z_{A, \infty} \phi=T_{A} \phi=0 \Longleftrightarrow{ }^{b} \bar{\nabla}^{A} \beta=0=\hat{\nabla}_{\zeta}^{A} \alpha \text { and }\left({ }^{b} \bar{\nabla}^{A}\right)^{*} \alpha=0=\hat{\nabla}_{\zeta}^{A} \beta . \\
\|\alpha\| \cdot\|\beta\|=0
\end{gathered}
$$

where the derivation $\hat{\nabla}_{\zeta}^{A}$ is the tensor product of the corresponding derivations on $\pi^{*} K_{\Sigma}^{ \pm 1 / 2}$ and $L_{N}$.

(c) $\frac{1}{2}\left(|\alpha|^{2}-|\beta|^{2}\right)=\mathrm{i} F_{A}\left(\zeta_{1}, \zeta_{2}\right)$.

We denote by $\mathcal{A}_{\infty}=\mathcal{A}_{\infty}\left(\ell, g, L_{N}\right)$ the collection of gauge equivalence classes of adiabatic solutions and we will call it the adiabatic moduli space. Note that it is not a priori clear whether any adiabatic solution is in fact an adiabatic limit. All we can state at this point is that

$$
\mathcal{S}_{\infty}:=\lim \mathcal{S}_{\delta} \subset \mathcal{A}_{\infty}
$$


We will further disect the adiabatic moduli space. It can be naturally decomposed into reducible and irreducible parts

$$
\mathcal{A}_{\infty}=\mathcal{A}_{\infty}^{\text {red }} \cup \mathcal{A}_{\infty}^{\text {irr }}:=\left\{(\phi, A) \in \mathcal{A}_{\infty} ; \phi=0\right\} \cup\left\{(\phi, A) \in \mathcal{A}_{\infty} ; \phi \neq 0\right\} .
$$

A. Reducible adiabatic solutions, i.e. $\|\alpha\|+\|\beta\|=0$. In this case the connection $A$ must be flat. Thus it is uniquely defined by its holonomy representation

$$
\operatorname{hol}_{A}: \pi_{1}\left(N_{\ell}\right) \rightarrow S^{1} .
$$

The fundamental group of $N_{\ell}$ can be presented as

$$
\pi_{1}\left(N_{\ell}\right)=\left\langle a_{1}, b_{1}, \ldots a_{g}, b_{g}, f \mid f^{-\ell} \cdot \prod_{i=1}^{g}\left[a_{i}, b_{i}\right]=\left[a_{j}, f\right]=\left[b_{k}, f\right]=1, \quad \forall j, k\right\rangle
$$

where $f$ denotes the homotopy class of a fiber. We conclude that the space of its $S^{1}$-representations is a $2 g+1$-dimensional torus if $\ell=0$ while if $\ell \neq 0$ it is a collection of $|\ell|$ tori $T^{2 g}$ parameterized by the group of $|\ell|$-th roots of unity. For any representation $\rho: \pi_{1}(N) \rightarrow S^{1}$ we have $\rho(f)=\exp (2 \pi k \mathrm{i} / \ell)$ for some $k \in \mathbb{Z}$ and if we denote by $L_{N, \rho}$ the line bundle it determines on $N$ we have

$$
c_{1}\left(L_{N, \rho}\right)=\hat{k} \in \mathbb{Z}_{|\ell|} \subset H^{2}\left(N_{\ell}, \mathbb{Z}\right) .
$$

To see this, denote by $A_{\rho}$ flat the connection on $L_{N, \rho}$ with holonomy $\rho$ and set $B_{\rho}:=A_{\rho}+\mathrm{i} k / \ell \eta$. Then the curvature of $B_{\rho}$ is purely horizontal, $F_{B_{\rho}}=\mathrm{i} k / \ell d \eta$ and its holonomy along the fibers is zero. Thus $B_{\rho}$ is the pull-back of a connection $D_{\rho}$ on a line bundle $L_{\Sigma, \rho} \rightarrow \Sigma$ whose curvature satisfies

$$
\pi^{*} F_{D_{\rho}}=\mathbf{i} k / \ell d \eta=-2 \mathbf{i} k *_{N} \eta=-2 \mathrm{i} k \pi^{*} d \operatorname{vol}_{\Sigma} .
$$

Thus

$$
c_{1}\left(D_{\rho}\right)=k / \pi d \operatorname{vol}_{\Sigma}
$$

i.e. $\operatorname{deg} L_{\Sigma, \rho}=k$ since by construction $\operatorname{vol}(\Sigma)=\pi$. The equality (3.20) now follows from the equality $L_{N, \rho}=\pi^{*} L_{\Sigma, \rho}$ combined with Gysin's exact sequence. To summarize, when $c_{1}\left(L_{N}\right)$ is a torsion class, the collection of gauge equivalence classes of reducible adiabatic solutions is a torus of dimension $2 g$.

Any reducible adiabatic solution is obviously a bona-fide solution of $S W_{\delta}$ for all $\delta>0$ and in particular it is a limit of (reducible) solutions of the 
Seiberg-Witten equations. Naturally one wonders whether there exists a sequence $\left(\phi_{\delta}, A_{\delta}\right)$ of irreducible solutions of $S W_{\delta}$ converging to a reducible adiabatic solution. Our next result will show this thing is not possible for nontrivial $\operatorname{spin}^{c}$ structures.

Proposition 3.9. Fix a $\operatorname{spin}^{c}$ structure on $N_{g, \ell}$ defined by a nontrivial line bundle $L_{N} \rightarrow N$ such that $c_{1}\left(L_{N}\right)$ is torsion. If $\left(\phi_{\delta}, A_{\delta}\right)$ is a sequence of solutions of $S W_{\delta}$ such that $A_{\delta}$ converges (in the sense of Theorem 3.2) to a flat connection then $\phi_{\delta} \equiv 0$ for all $\delta \gg 1$.

Proof. Assume the contrary. Thus, for all $\delta \gg 1$ there exists $\left(A_{\delta}, \phi_{\delta}\right) \in \mathcal{S}_{\delta}$ such that $\phi_{\delta} \neq 0$. Set $\psi_{\delta}=\frac{1}{\left\|\phi_{\delta}\right\|} \phi_{\delta}$ so that $\left\|\psi_{\delta}\right\|=1, \forall \delta$. We can now apply the adiabatic decoupling lemma to the sequence $\left(A_{\delta}, \psi_{\delta}\right)$ to conclude that there exists $\psi \in L^{1,2}\left(\mathbb{S}_{L}\right)$ such that $\|\psi\|=1$ and

$$
Z_{A, \infty} \psi=T_{A} \psi=0
$$

where $A=\lim A_{\delta}$. Thus $\psi$ is a nontrivial section of $\pi^{*}\left(K_{\Sigma}^{-1 / 2} \oplus K_{\Sigma}^{1 / 2}\right) \otimes$ $L$ which is covariant constant along the fibers of $N$ with respect to the connection $\left(\nabla^{\infty,-} \oplus \nabla^{\infty,+}\right) \otimes A$. The holonomy of $\nabla^{\infty}$ along a fiber is trivial (since it is a pullback connection) and since $c_{1}(L) \neq 0$ the holonomy of the flat connection $A$ around a fiber is nontrivial. Hence there cannot exist nontrivial, fiberwise covariant constant sections of $\mathbb{S}_{L}$. This contradiction concludes the proof of Proposition 3.9.

B. Irreducible adiabatic solutions, i.e. $\|\alpha\|+\|\beta\| \neq 0$.

Lemma 3.10. If $\|\alpha\|+\|\beta\| \neq 0$ then (modulo gauge transformations) the connection $A$ is the pull back of a connection on a line bundle $L_{\Sigma} \rightarrow \Sigma$.

Proof of the lemma. Because the connection $A$ is vertically flat one can prove easily that the holonomy along a fiber is independent of the particular fiber. Since either $\alpha$ or $\beta$ is not identically zero and is covariant constant along fibers we deduce that the holonomy along fibers is trivial.

Consider now an irreducible adiabatic solution $(\alpha \oplus \beta, A)$. Thus there exists a connection $A_{\Sigma}$ on $L_{\Sigma}$ such that $\pi^{*} L_{\Sigma}=L_{N}$ and $\pi^{*} A_{\Sigma}=A$. Since 
$\alpha$ and $\beta$ are covariant constant along fibers they can be regarded as sections of $L_{\Sigma} \otimes K_{\Sigma}^{ \pm 1 / 2}$. We can decide which of $\alpha$ or $\beta$ vanishes. More precisely if

$$
\operatorname{deg} L_{\Sigma}<\operatorname{deg} K_{\Sigma}^{-1 / 2}=1-g
$$

then the line bundle $L_{\Sigma} \otimes K_{\Sigma}^{1 / 2}$ cannot admit holomorphic sections so that $\beta \equiv 0$. In particular, $\alpha \not \equiv 0$. In this case, using the equality (3.2) we deduce after an integration along fibers that

$$
1-g>\operatorname{deg} L_{\Sigma}=\frac{\mathrm{i}}{2 \pi} \int_{N} \eta \wedge F_{A}=\frac{1}{2}\|\alpha\|^{2} .
$$

This is impossible since $\alpha \neq 0$. If

$$
\operatorname{deg} L_{\Sigma}>\operatorname{deg} K_{\Sigma}^{1 / 2}=g-1
$$

then $L_{\Sigma} \otimes K_{\Sigma}^{-1 / 2}$ cannot admit antiholomorphic sections so that $\alpha \equiv 0$. Reasoning as above we deduce another contradiction so we can conclude the adiabatic limit set is empty when $\left|\operatorname{deg} L_{\Sigma}\right|>g-1$.

We now analyze what happens when $\left|\operatorname{deg} L_{\Sigma}\right| \leq g-1$. We will discuss only the case $1-g \leq \operatorname{deg} L_{\Sigma} \leq 0$. The other half is completely similar.

Using the equality (3.2) again we deduce

$$
\frac{1}{4 \pi}\left(\|\alpha\|^{2}-\|\beta\|^{2}\right)=\operatorname{deg} L_{\Sigma} \leq 0 .
$$

Since one of $\alpha$ or $\beta$ is identically zero we deduce that $\alpha \equiv 0$. Hence $\beta$ is a nontrivial holomorphic section of $L_{\Sigma} \otimes K_{\Sigma}^{1 / 2}$. Moreover, $\operatorname{deg} L_{\Sigma} \neq 0$. This shows that when $g=1$, for any $\operatorname{spin}^{c}$ structure, the corresponding $S W$ equations have no irreducible adiabatic solutions.

We see that the irreducible part of the adiabatic limit set is determined entirely in terms of of objects defined on the base of $N$. Using the terminology of $[\mathrm{Br}]$ (or [BGP]), the pair $\left(\phi_{\Sigma}, A_{\Sigma}\right)$ is a vortex pair on $L_{\Sigma} \rightarrow \Sigma$. Furthermore, in $[\mathrm{Br}]$ it is shown that the correspondence $\phi \mapsto\left\{\phi^{-1}(0)\right\}$ (multiplicities included) identifies the moduli space of gauge equivalence classes of vortex pairs on $L_{\Sigma}$ with a symmetric product $\Sigma(d)$ of $d$ copies of $\Sigma, d=(g-1)-\left|\operatorname{deg} L_{\Sigma}\right|$ (see also the discussion in $\S 4.2$.)

When $\operatorname{deg} L_{\Sigma}=1-g$ the bundle $L_{\Sigma} \otimes K_{\Sigma}^{1 / 2}$ is topologically trivial. The only topologically trivial holomorphic line bundle which admits a holomorphic section is the holomorphically trivial line bundle. Hence, when $\operatorname{deg} L_{\Sigma}=1-g$ there exists exactly one irreducible adiabatic solution (up to a gauge transformations). 
The above picture resembles very much the exact computations in the trivial case $\ell=0$. There are however some notable differences. When $\ell=0$ if the $\operatorname{spin}^{c}$ structure is non-trivial there exist no reducible solutions for the simple fact that there exist no flat connections. This is no longer the case when $\ell \neq 0$. Moreover, according to Gysin's exact sequence the kernel of the morphism

$$
\pi^{*}: \mathbb{Z} \cong H^{2}(\Sigma, \mathbb{Z}) \rightarrow H^{2}\left(N_{\ell}, \mathbb{Z}\right)
$$

is the subgroup $\ell \mathbb{Z}$. If $\ell=0$ this means the $\operatorname{spin}^{c}$-structure $L_{N}$ uniquely determines a line bundle $L_{\Sigma} \rightarrow \Sigma$ such that $\pi^{*} L_{\Sigma} \cong L_{N}$.

If $\ell \neq 0$ there are infinitely many line bundles on $\Sigma$ with the above property and their degrees are congruent modulo $\ell$ with $c_{1}\left(L_{N}\right) \in \mathbb{Z}_{\ell} \subset$ $H^{2}\left(N_{\ell}, \mathbb{Z}\right)$. On the other hand, in case $\mathbf{B}$ the only line bundles on $\Sigma$ relevant in the adiabatic limit are those of degree in the interval $[-(g-1),(g-1)]$.

Assume now $|\ell| \geq 2 g$ and

$$
c_{1}\left(L_{N}\right) \in \mathcal{V}_{g, \ell}=\{g, g+1, \cdots,|\ell|-g\}(\bmod \ell) \subset \mathbb{Z}_{\ell}
$$

Then there is no integer $n$ such that $n \bmod \ell \in \mathcal{V}_{g, \ell}$ and $0<|n| \leq g-1$. In other words if $c_{1}\left(L_{N}\right) \in \mathcal{V}_{g, \ell}$ then there exists no line bundle $L_{\Sigma} \rightarrow \Sigma$ of degree $0<\left|\operatorname{deg} L_{\Sigma}\right| \leq g-1$ such that $\pi^{*} L_{\Sigma}=L_{N}$. We have thus proved the following result.

Corollary 3.11. (a) If $|\ell| \geq 2 g \geq 4$ and $L_{N} \rightarrow N_{\ell}$ is a line bundle on $N_{\ell}$ with $c_{1}\left(L_{N}\right) \in \mathcal{V}_{g, \ell}$ then the collection $\mathcal{A}_{\infty}^{\text {irr }}=\emptyset$.

(b) If $g=1,|\ell| \geq 1$ and $L$ is nontrivial, then the set $\mathcal{A}_{\infty}=\emptyset$. If $L$ is trivial then $\mathcal{A}_{\infty}^{\text {irr }}=\emptyset$.

The range $\mathcal{V}_{g, \ell}$ of $\operatorname{spin}^{c}$ structures will be called the adiabatically stable range. Using Proposition 3.9 we deduce the following consequence.

Corollary 3.12. For any $\operatorname{spin}^{c}$ structure in the stable range the corresponding $S W_{\delta}$ equations have no irreducible solutions for $\delta \gg 1$.

Remark 3.13. The above arguments extend easily to most Seifert manifolds. More precisely, as indicated in [FS], many Seifert manifolds are finite quotients of circle bundles. One can easily incorporate a finite action in the above arguments. The adiabatic solutions on such a Seifert manifold are then invariant adiabatic solutions on a circle bundle. This can be conveniently reformulated using the language of orbifolds as in [MOY]. 


\section{Variational features of the adiabatic moduli space.}

In this section we prove that the irreducible components of the adiabatic moduli space (over a smooth $S^{1}$-fibration) are Bott nondegenerate as critical sets of a suitable energy functional.

\subsection{Algebraic and analytic preliminaries.}

We continue to use the same notations as in §3.3. Fix a line bundle $L_{\Sigma} \rightarrow \Sigma$ and set $L=\pi^{*} L_{\Sigma} \rightarrow N$.

The configuration space for all the $S W$ equations will be

$$
\mathcal{C}=\mathfrak{A}^{1,2}(L) \oplus L^{1,2}\left(S_{L}\right)
$$

consisting of pairs of $L^{1,2}$-connections and spinors. This is a metric affine space but the $L^{1,2}$-metric obviously depends on the Riemann metric on $N$. When using the deformed metric $g_{\delta}$ we will write $\mathcal{C}_{\delta}$ instead.

This configuration space is acted upon by the gauge group $\mathfrak{G}_{L}$ consisting of $L^{2,2}$-gauge transformations of $L$. For each $\mathfrak{c}=(\phi, A)$ we will denote by $[c]=[\phi, A]$ its gauge equivalence class.

Using Lemma 3.1 we deduce the solutions of $(S W)_{\infty}$ are critical points of the energy functional

$$
\mathfrak{E}(\phi, A)=\mathfrak{f}(\phi, A)-\frac{\lambda}{4}\|\phi\|^{2} .
$$

This energy functional depends on the metric $g_{\delta}$ and we will indicate this dependence by a $\delta$-subscript. (We will use the same convention when indicating various Sobolev norms.) However, its critical set which is $\mathcal{A}_{\infty}$ does not depend on $\delta$. Lemma 3.1 shows that the $L^{2}$-gradient of $\mathfrak{E}_{\delta}$ is

$$
\left.\left.\nabla \mathfrak{E}_{\delta}\right|_{(\psi, A)}=\left(\mathfrak{D}_{A}^{\delta}-\lambda_{\delta} / 2\right) \psi \oplus \mathbf{c}_{\delta}^{-1}\left(\tau_{\delta}(\psi)\right)-* F_{A}\right) .
$$

Since $c_{1}(L)$ is torsion the functional $\mathfrak{E}_{\delta}$ is $\mathfrak{G}$-invariant and thus descends to a well defined functional $\underline{\mathfrak{E}}_{\delta}$ on

$$
\mathcal{B}_{\delta}:=\mathcal{C}_{\delta} / \mathfrak{G}
$$

We will be interested in its restriction to the irreducible part

$$
\mathcal{B}_{\delta}^{*}=\{[\phi, A] ; \phi \neq 0\}
$$

which is a Hilbert manifold (see e.g. [FU]). The following is the main result of this section. 
Theorem 4.1. For $\delta \gg 1$ the energy functional $\underline{\mathfrak{E}}_{\delta}$ is Bott nondegenerate.

To prove the Bott nondegeneracy of $\underline{\mathfrak{E}}_{\delta}$ we have to establish two things.

(a) $\mathcal{A}_{\infty}^{*}$ is a smooth manifold and

(b) the Hessian of $\underline{\mathfrak{E}}_{\delta}$ along $\mathcal{A}_{\infty}^{*}$ is nondegenerate in the normal directions.

The first task is to describe a suitable functional set-up and to keep track of the gauge action. We will work with the undeformed metric $g$ on $N$ leaving the reader to perform the obvious changes to $g_{\delta}$. Also, when speaking of adjoints of operators we will always understand $L^{2}$-adjoints.

The group $\mathfrak{G}$ is a Hilbert Lie group (cf. [FU]) and its Lie algebra can be identified with the space $\mathfrak{g}:=L^{2,2}(N, \mathbf{i} \mathbb{R})$. The exponential map has the form

$$
\mathfrak{g} \ni \mathbf{i} f \mapsto\left(\exp (\mathbf{i} f): N \rightarrow S^{1}\right) .
$$

The tangent space to the orbit $\mathfrak{O}_{\phi, A}$ of $\mathfrak{G}$ through $(\phi, A)$ is the range of the infinitesimal action operator

$$
\mathfrak{L}=\mathfrak{L}_{\phi, A}: \mathfrak{g} \rightarrow \mathfrak{X}:=L^{1,2}\left(\mathbb{S}_{L}\right) \oplus L^{1,2}\left(\mathbf{i} T^{*} N\right), \quad \text { if } \mapsto \mathbf{i} f \phi \oplus(-\mathbf{i} d f) .
$$

The tangent space to $\mathcal{B}^{*}$ at $[\phi, A]$ can be identified with the $L^{2}$-orthogonal complement to $T_{\phi, A} \mathfrak{O}_{\phi, A}$ in $\mathfrak{X}$ and ultimately, with the kernel of $\mathfrak{L}^{*}$, the adjoint of $\mathfrak{L}$. We will treat $\mathfrak{X}$ as a real Hilbert space and we will generically denote its elements by $(\dot{\psi}, \mathrm{i} \dot{a})$ where $\dot{a}$ denotes a real valued 1 -form. The adjoint of $\mathfrak{L}$ is determined from the equality

$$
\int_{N}\langle\mathfrak{L} \mathbf{i} f, \dot{\psi} \oplus \mathbf{i} \dot{a}\rangle d v_{g}=\int_{N} f \cdot \mathfrak{R e} \mathfrak{L}^{*}(\dot{\psi} \oplus \mathbf{i} \dot{a}) d v_{g}, \quad \forall f, \dot{\psi}, \mathbf{i} \dot{a}
$$

upon integrating by parts in the left-hand-side. A simple computation yields

$$
\mathfrak{L}^{*}(\dot{\psi} \oplus \mathbf{i} \dot{a})=-\mathbf{i} d^{*} \dot{a}-\mathbf{i} \mathfrak{I m}\langle\phi, \dot{\psi}\rangle \in L^{2}(N, \mathbf{i} \mathbb{R})
$$

where $\langle\cdot, \cdot\rangle$ denotes the complex, pointwise inner product in the fibers of $\mathbb{S}_{L}$.

The affine structure on $\mathcal{C}$ can be used to linearize $\nabla \mathfrak{E}$ at a given configuration $(\phi, A)$. More precisely, define the unrestricted Hessian at $(\phi, A)$ as the operator

$$
\mathfrak{H}=\mathfrak{H}_{\phi, A}: \mathfrak{X} \rightarrow \mathfrak{Y}:=L^{2}\left(\mathbb{S}_{L}\right) \oplus L^{2}\left(\mathbf{i} T^{*} N\right)
$$

defined by

$$
\mathfrak{H}(\dot{\psi}, \mathbf{i} \dot{a})=\left.\frac{d}{d t}\right|_{t=0} \nabla \mathfrak{E}(\phi+t \dot{\psi}, A+t \mathbf{i} \dot{a})
$$


A simple computation yields

$$
\mathfrak{H}\left[\begin{array}{c}
\dot{\psi} \\
\mathbf{i} \dot{a}
\end{array}\right]=\left[\begin{array}{c}
\mathfrak{D}_{A} \dot{\psi}+\mathbf{c}(\mathbf{i} \dot{a}) \phi-\lambda \dot{\psi} / 2 \\
-\mathbf{i} * d \dot{a}+\dot{\tau}(\phi, \dot{\psi})
\end{array}\right]
$$

The term $\dot{\tau}(\phi, \dot{\psi})$ needs further clarification. It is formally defined by the equality

$$
\dot{\tau}(\phi, \dot{\psi}):=\left.\frac{d}{d t}\right|_{t=0} \tau(\phi+t \dot{\psi})
$$

where we regard $\tau$ as a quadratic map $\tau: \mathbb{S}_{L} \rightarrow \mathbf{i} T^{*} N$. Using the local description

$$
\tau(\phi)=\frac{1}{2} \sum_{i=0}^{2}\left\langle\phi, \mathbf{c}\left(\eta_{i}\right) \phi\right\rangle \eta_{i}
$$

we deduce after some simple manipulations that

$$
\dot{\tau}(\phi, \dot{\psi})=\mathrm{i} \mathfrak{I m} \sum_{i=0}^{2}\left\langle\phi, \mathbf{c}\left(\eta_{i}\right) \dot{\psi}\right\rangle \eta_{i} .
$$

Sometimes it will be convenient to regard $\dot{\tau}$ as a symmetric endomorphism of $\mathbb{S}_{L}$. To obtain its explicit description we must derivate the equality

$$
\tau(\phi)=\bar{\phi} \otimes \phi-|\phi|^{2} / 2
$$

along $\dot{\psi}$ and we get

$$
\dot{\tau}(\phi, \dot{\psi})=\dot{\bar{\psi}} \otimes \phi+\bar{\phi} \otimes \dot{\psi}-\mathfrak{R e}\langle\phi, \dot{\psi}\rangle .
$$

The affine connection on $\mathcal{C}^{*}$ we used to define the Hessian of $\mathfrak{E}$ is $\mathfrak{G}$ equivariant and thus defines a connection on the quotient $\mathcal{B}^{*}$ which can be used to define the Hessian $\underline{\mathfrak{H}}$ of $\underline{\mathfrak{E}}$. This leads to the following formula for $\underline{\mathfrak{H}}_{\phi, A}$

$$
\left.\underline{\mathfrak{H}}_{\phi, A}(\dot{\psi}, \mathbf{i} \dot{a})=\mathfrak{P r o j}\right)_{\phi, A} \mathfrak{H}(\dot{\psi}, \mathbf{i} \dot{a}), \quad \forall(\dot{\psi}, \mathbf{i} \dot{a}) \in \operatorname{ker} \mathfrak{L}_{\phi, A}^{*}
$$

where $\mathfrak{P r o j}_{\phi, A}$ denotes the $L^{2}$-orthogonal projection onto the kernel of $\mathfrak{L}_{\phi, A}^{*}$.

We will be interested only in the eigenvalues of $\underline{\mathfrak{I}}_{\phi, A}$. In searching for eigenvectors and eigenvalues we will use a well known trick in gauge theory. A pair $(\dot{\psi}, \mathbf{i} \dot{a}) \in \operatorname{ker} \mathfrak{L}^{*}$ is an eigenvector of $\underline{\mathfrak{H}}$ corresponding to the eigenvalue $\mu$ if and only if there exists an infinitesimal gauge transformation if $\in \mathfrak{g}$ such that the triple $(\dot{\psi}, \mathbf{i} \dot{a}, \mathbf{i} f)$ is a solution of the following system of p.d.e.s

$$
\hat{\mathfrak{H}}_{\phi, A}\left[\begin{array}{c}
\dot{\psi} \oplus \mathbf{i} \dot{a} \\
\mathbf{i} f
\end{array}\right]:=\left[\begin{array}{cc}
\mathfrak{H} & \mathfrak{L} \\
\mathfrak{L}^{*} & 0
\end{array}\right]\left[\begin{array}{c}
\dot{\psi} \oplus \mathbf{i} \dot{a} \\
\mathbf{i} f
\end{array}\right]=\mu\left[\begin{array}{c}
\dot{\psi} \oplus \mathbf{i} \dot{a} \\
0
\end{array}\right]
$$


$\hat{\mathfrak{H}}$ is called the stabilized hessian of $\mathfrak{E}$ at $(\phi, A)$. A solution of the above equation will be called a $\mu$-eigenvector of $\hat{\mathfrak{H}}$. The proof of following result is left to the reader.

Lemma 4.2. The partial differential operator $\hat{\mathfrak{H}}_{\phi, A}$ is elliptic and selfadjoint for all $(\phi, A) \in \mathcal{A}_{\infty}^{*}$.

We conclude this subsection with a variational identity which will play a key role in the sequel. Set

$$
\mathfrak{Z}:=\mathfrak{X} \oplus L^{1,2}(N, \mathbf{i} \mathbb{R}) .
$$

Lemma 4.3 (Main variational identity). $F i x(\phi, A) \in \mathcal{A}_{\infty}^{*}$. If

$$
(\dot{\psi}, \mathbf{i} \dot{a}, \mathbf{i} f) \in \mathfrak{Z}
$$

is a $\mu$-eigenvector of $\hat{\mathfrak{H}}_{\phi, A}$ then for all $\dot{\phi} \in L^{1,2}\left(\mathbb{S}_{L}\right)$ we have

$$
\begin{aligned}
\mathfrak{R e}\left(\int_{N}\right. & \left.\left\langle\mathbf{D}_{A} \dot{\psi}, \mathbf{D}_{A} \dot{\phi}\right\rangle d v_{g}+\int_{N}\langle\dot{\psi}, \dot{\phi}\rangle|\phi|^{2} d v_{g}\right) \\
& -\mathfrak{R e}\left(\int_{N}\left\langle 2 \mathbf{i} \nabla_{\dot{a}} \phi+\nu \mathbf{c}(\mathbf{i} \dot{a}) \phi, \dot{\phi}\right\rangle d v_{g}\right)-\frac{\lambda}{2} \mathfrak{I m}\left(\int_{N} f\langle\phi, \dot{\phi}\rangle d v_{g}\right) \\
& =\mathfrak{R e}\left(\int_{N}(\mu-\lambda / 2)\left\langle\mathbf{D}_{A} \dot{\psi}, \dot{\phi}\right\rangle+\frac{\lambda \mu}{2}\langle\dot{\psi}, \dot{\phi}\rangle d v_{g}\right) .
\end{aligned}
$$

where $\nu=\mu+\lambda / 2, \mathbf{D}_{A}$ is the adiabatic Dirac operator $\mathbf{D}_{A}=Z_{A, \infty}+T_{A}$, while $\nabla_{\dot{a}}=\nabla_{\dot{a}}^{A}$ denotes the covariant derivative on $\mathbb{S}_{L}$ along the vector field dual to $\dot{a}$.

Proof. The equality (4.5) has the more explicit form

$$
\begin{cases}\mathfrak{D}_{A} \dot{\psi}+\mathbf{c}(\dot{\mathbf{i}} \dot{a}) \phi-\lambda \dot{\psi} / 2+\mathbf{i} f \phi & =\mu \dot{\psi} \\ -\mathbf{i} * d \dot{a}+\dot{\tau}(\phi, \dot{\psi})-\mathbf{i} d f & =\mu \mathbf{i} \dot{a} \\ d^{*} \dot{a} & =\mathfrak{I m}\langle\dot{\psi}, \phi\rangle\end{cases}
$$

Now apply $\mathfrak{D}_{A}$ to the first equation in (4.6). We deduce

$$
\mathfrak{D}_{A}^{2} \dot{\psi}+\mathfrak{D}_{A} \mathbf{c}(\mathbf{i} \dot{a}) \phi+\mathfrak{D}_{A}(\mathbf{i} f \phi)=\nu \mathfrak{D}_{A} \dot{\psi} .
$$

We can transform the last term in the left-hand-side of (4.7) using the classical identity $\mathfrak{D}_{A} \mathbf{i} f \phi=\mathbf{c}(\mathbf{i} d f) \phi+\mathbf{i} f \mathfrak{D}_{A} \phi$. Similarly since $\mathfrak{D}_{A} \mathbf{c}(\mathbf{i} \dot{a})=$ $-\mathbf{c}(\mathbf{i} \dot{a}) \mathfrak{D}_{A}+\left\{\mathfrak{D}_{A}, \mathbf{c}(\mathbf{i} \dot{a})\right\}$ we deduce from Proposition 3.45 of $[\mathrm{BGV}]$ that

$$
\mathfrak{D}_{A} \mathbf{c}(\mathbf{i} \dot{a})=-\mathbf{c}(\mathbf{i} \dot{a}) \mathfrak{D}_{A}-2 \mathbf{i} \nabla_{\dot{a}}+\mathbf{c}(\mathbf{i} * d \dot{a})+\mathbf{i} d^{*} \dot{a}
$$


Recall now that $\mathfrak{D}_{A} \phi=\lambda \phi / 2$ since $(\phi, A) \in \mathcal{A}_{\infty}$. Puting all these facts together in (4.7) we deduce

$$
\begin{aligned}
\mathfrak{D}_{A}^{2} \dot{\psi}+\left\{-\lambda \mathbf{c}(\mathbf{i} \dot{a}) / 2-2 \mathbf{i} \nabla_{\dot{a}}+\right. & \mathbf{c}(\mathbf{i} * d \dot{a}) \\
& \left.+\mathbf{c}(\mathbf{i} d f)+\mathbf{i}\left(d^{*} \dot{a}+\lambda f / 2\right)\right\} \phi=\nu \mathfrak{D}_{A} \dot{\psi} .
\end{aligned}
$$

The second equation in (4.6) gives $\mathbf{c}(\mathbf{i} * d \dot{a})+\mathbf{c}(\mathbf{i} d f)=\dot{\tau}(\phi, \dot{\psi})-\mu \mathbf{c}(\mathbf{i} \dot{a})$ while the third one implies $\left(d^{*} a\right) \phi=(\mathfrak{I m}\langle\dot{\psi}, \phi\rangle) \phi$. Thus we can rephrase (4.8) as

$$
\mathfrak{D}_{A}^{2} \dot{\psi}-\nu \mathbf{c}(\mathbf{i} \dot{a}) \phi+\dot{\tau}(\phi, \dot{\psi}) \phi+\mathbf{i}(\mathfrak{I m}\langle\dot{\psi}, \phi\rangle+\lambda f / 2) \phi=\nu \mathfrak{D}_{A} \dot{\psi}
$$

Using the equality $\mathfrak{D}_{A}=\mathbf{D}_{A}+\lambda / 2$ we deduce $\mathfrak{D}_{A}^{2}=\mathbf{D}_{A}^{2}+\lambda \mathbf{D}_{A}+\lambda^{2} / 4$ and (4.9) can be further transformed to

$$
\begin{aligned}
\mathbf{D}_{A}^{2} \dot{\psi}-\nu \mathbf{c}(\mathbf{i} \dot{a}) \phi+\dot{\tau}(\phi, \dot{\psi}) \phi+\mathbf{i}(\mathfrak{I m}\langle\dot{\psi}, \phi\rangle+\lambda f / 2) \phi \\
=\left\{(\mu-\lambda / 2) \mathbf{D}_{A}+\lambda \mu / 2\right\} \dot{\psi}
\end{aligned}
$$

Using (4.4) we deduce that

$$
\dot{\tau}(\phi, \dot{\psi}) \phi=\langle\phi, \dot{\psi}\rangle \phi+\langle\phi, \phi\rangle \dot{\psi}-\mathfrak{R e}\langle\phi, \dot{\psi}\rangle \phi
$$

Hence we have the pointwise equalities

$$
\begin{aligned}
\mathfrak{R e}\langle\dot{\tau}(\phi, \dot{\psi}) \phi, \dot{\phi}\rangle & =\mathfrak{R e}\left(\langle\phi, \dot{\psi}\rangle\langle\phi, \dot{\phi}\rangle+|\phi|^{2}\langle\dot{\psi}, \dot{\phi}\rangle-(\mathfrak{R e}\langle\phi, \dot{\psi}\rangle)\langle\phi, \dot{\phi}\rangle\right) \\
& =-\mathfrak{I m}\langle\phi, \dot{\psi}\rangle \cdot \mathfrak{I m}\langle\phi, \dot{\phi}\rangle+|\phi|^{2} \mathfrak{R e}\langle\dot{\psi}, \dot{\phi}\rangle .
\end{aligned}
$$

Similarly we get

$$
\mathfrak{R e}\langle\mathfrak{i} \mathfrak{I m}\langle\dot{\psi}, \phi\rangle \phi, \dot{\psi}\rangle=\mathfrak{I m}\langle\phi, \dot{\psi}\rangle \cdot \mathfrak{I m}\langle\phi, \dot{\phi}\rangle
$$

Hence

$$
\mathfrak{R e}\{\langle\dot{\tau}(\phi, \dot{\psi}) \phi, \dot{\phi}\rangle+\langle\mathbf{i} \mathfrak{I m}\langle\dot{\psi}, \phi\rangle \phi, \dot{\psi}\rangle\}=|\phi|^{2} \mathfrak{R e}\langle\dot{\psi}, \dot{\phi}\rangle .
$$

If we now take the real inner product of (4.10) by $\dot{\phi}$, then integrate by parts and use (4.11) we obtain the main variational identity. 


\subsection{Abelian vortices on Riemann surfaces.}

In this subsection we describe a natural smooth structure on $\mathcal{A}_{\infty}^{*}$. We begin by considering a related gauge theoretic problem on a Riemann surface $\Sigma$ of positive genus $g$. Fix a Riemann metric $h$ on $\Sigma$ and denote by $K$ the canonical line bundle with the induced metric and connection. Consider a hermitian line bundle $L \rightarrow \Sigma$

An $L$-valued vortex pair on $\Sigma$ is a pair $(\phi, A)$ where $\phi=\alpha \oplus \beta$ is a section of $\mathbb{S}_{L}:=L \otimes K^{-1 / 2} \oplus L \otimes K^{1 / 2}$ and $A$ is a hermitian connection on $L$ satisfying the vortex equations

$$
\begin{gathered}
{\left[\begin{array}{cc}
0 & \bar{\partial}_{A} \\
\bar{\partial}_{A}^{*} & 0
\end{array}\right] \cdot\left[\begin{array}{l}
\alpha \\
\beta
\end{array}\right]=\left[\begin{array}{l}
0 \\
0
\end{array}\right]} \\
\mathbf{i} * F_{A}=\frac{1}{2}\left(|\alpha|^{2}-|\beta|^{2}\right), \quad\|\alpha\| \cdot\|\beta\|=0 .
\end{gathered}
$$

The solutions of this equation were described in great detail in [Br]. Here we want to prove some additional facts related to the deformation complex of this problem. For brevity we will consider only the holomorphic case $\beta \neq 0$ since the anti-holomorphic situation can be dealt with following a similar approach. Thus $(4.12)+(4.13)$ can be rephrased as

$$
\bar{\partial}_{A} \beta=0, \quad * F_{A}=-\frac{\mathbf{i}}{\mathbf{2}}|\beta|^{2} .
$$

Note that in the holomorphic case the second equation implies $\operatorname{deg} L \leq 0$. The functional set-up is identical to the one described in $\S 4.1$ for the SeibergWitten equations and we will omit references to various Sobolev spaces to make the presentation more transparent.

The space of configurations $\mathcal{C}=\Gamma\left(L \otimes K^{1 / 2}\right) \oplus \mathfrak{A}(L)$ is acted upon by the gauge group Aut $(L)$ whose Lie algebra is $i \Omega^{0}(\Sigma)$. The infinitesimal action of $\mathbf{i} f \in \mathbf{i} \Omega^{0}(\Sigma)$ on $(\beta, A)$ is given as usual by

$$
(\beta, A) \stackrel{I_{\beta, A}}{\mapsto}(\mathrm{i} f \beta, A-\mathbf{i} d f) .
$$

Denoting the tangent spaces to $\mathcal{C}$ by $\dot{\mathcal{C}}$ we can compute the linearization $\Upsilon=\Upsilon_{\beta, A}: \dot{\mathcal{C}} \rightarrow \Gamma\left(L \otimes K^{-1 / 2}\right) \oplus \mathbf{i} \Omega^{0}(\Sigma)$ of (4.14) and we get

$$
\Upsilon\left[\begin{array}{c}
\dot{\beta} \\
\mathbf{i} \dot{a}
\end{array}\right]=\left[\begin{array}{c}
\bar{\partial}_{A} \dot{\beta}+2^{-1 / 2} \mathbf{i} \dot{a}^{0,1} \beta \\
\mathbf{i} * d \dot{a}-\mathbf{i} \mathfrak{R e}\langle\beta, \dot{\beta}\rangle
\end{array}\right]
$$


$\mathrm{i} \dot{a}^{0,1}$ component is the $K^{-1}$-component of $\mathrm{i} \dot{a}$ corresponding to the orthogonal decomposition $T^{*} \Sigma \otimes \mathbb{C} \cong K \oplus K^{-1}$. The normalization constant $2^{-1 / 2}$ is obtained from the equality $(d f)^{0,1}=2^{1 / 2} \bar{\partial} f$. We will sometimes find it convenient to write $P^{0,1} \dot{a}$ instead of $\dot{a}^{0,1}$.

The deformation complex of this problem is the following sequence of partial differential operators

$$
\begin{aligned}
\left(V_{\beta, A}\right): 0 \rightarrow \mathbf{i} \Omega^{0}(\Sigma) \stackrel{I}{\rightarrow} \Gamma\left(L \otimes K^{1,2}\right) \oplus & \stackrel{\mathbf{i} \Omega^{1}(\Sigma)}{ } \\
& \stackrel{\Upsilon}{\rightarrow} \Gamma\left(L \otimes K^{-1 / 2}\right) \oplus \mathbf{i} \Omega^{0}(\Sigma) \rightarrow 0 .
\end{aligned}
$$

Let us first notice the following fact.

Lemma 4.4. If $(\beta, A)$ is a solution of (4.14) with $\beta \neq 0$ then

(a) $\left(V_{\beta, A}\right)$ is an elliptic complex.

(b) $H^{0}\left(V_{\beta, A}\right)=H^{2}\left(V_{\beta, A}\right)=0$.

(c) $\operatorname{dim}_{\mathbb{R}} H^{1}\left(V_{\beta, A}\right)=2 d_{+}(L):=2(g-1+\operatorname{deg} L)$.

Proof. (a) The fact that $\left(V_{\beta, A}\right)$ is a complex (i.e. $\Upsilon \circ I=0$ ) is elementary and is left to the reader. The ellipticity follows by observing that modulo zero order terms this complex is the direct sum of the Hodge-DeRham complex on $\Lambda^{*} T^{*} \Sigma$ and the (degree shifted) Hodge-Dolbeault complex corresponding to the line bundle $L \otimes K^{1 / 2}$. In particular we have the following equality of Euler characteristics (over $\mathbb{R}$ )

$$
\chi(V)=\chi(\Sigma)-\chi\left(L \otimes K^{1 / 2}\right)=2(1-g-\operatorname{deg} L) .
$$

(b) Since the infinitesimal action of $\operatorname{Aut}(L)$ on $(\beta, A)$ is free $(\beta \neq 0)$ we deduce $H^{0}(V)=0$. To prove that $H^{2}\left(V_{\beta, A}\right)=0$ we rely on the ellipticity of the complex and it suffices to show $\operatorname{ker} \Upsilon^{*}=0$. Let $(\alpha, \mathbf{i} f) \in \operatorname{ker} \Upsilon^{*}$ that is

$$
\int_{\Sigma} \mathfrak{R e}\langle\Upsilon(\dot{\beta} \oplus \mathbf{i} \dot{a}), \alpha \oplus \mathrm{i} f\rangle d v_{h}=0 \quad \forall \dot{\beta} \oplus \mathbf{i} \dot{a} \in \dot{\mathcal{C}}_{\beta, A} .
$$

Several clever choices of $\dot{\beta}$ and $\mathbf{i} \dot{a}$ in (4.16) will produce the desired conclusion. Let us now describe these choices.

- $\dot{\beta}=\bar{\partial}_{A}^{*} \alpha, \dot{a}=0$. We get

$$
\int_{\Sigma}\left|\bar{\partial}_{A}^{*} \alpha\right|^{2} d v_{h}=\int_{\Sigma} \mathfrak{R e}\left\langle\bar{\partial}_{A}^{*} \alpha, f \beta\right\rangle d v_{h}
$$


and integrating by parts in the right hand side we get

$$
\int_{\Sigma}\left|\bar{\partial}_{A}^{*} \alpha\right|^{2} d v_{h}=\int_{\Sigma} \mathfrak{R e}\langle\alpha,(\bar{\partial} f) \beta\rangle d v_{h}
$$

- $\dot{\beta}=0, \dot{a}=* d f$. We get

$$
2^{-1 / 2} \int_{\Sigma} \mathfrak{R e}\left\langle\mathbf{i} P^{0,1}(* d f) \beta, \alpha\right\rangle d v_{h}=\int_{\Sigma}|d f|^{2} d v_{h} .
$$

The term in the left-hand-side needs further clarification. We will work out the expression of the integrand in local conformal coordinates $(x, y)$ on $\Sigma$. Set $z=x+\mathrm{i} y$. We have $* d f=f_{x} d y-f_{y} d x$. Since $d x=(d z+d \bar{z}) / 2$ and $d y=-\mathbf{i}(d z-d \bar{z}) / 2$ we deduce

$$
2^{-1 / 2} P^{0,1}(* d f)=\mathrm{i} \bar{\partial} f .
$$

This yields

$$
-\mathfrak{R e} \int_{\Sigma}\langle(\bar{\partial} f) \beta, \alpha\rangle d v_{h}=\int_{\Sigma}|d f|^{2} d v_{h}
$$

Coupling (4.17) and (4.18) we deduce $d f \equiv 0$ and $\bar{\partial}_{A}^{*} \alpha \equiv 0$.

- $\dot{\beta}=0, \dot{a}$ arbitrary. Since $d f=0$ we deduce

$$
\int_{\Sigma} \mathfrak{R e}\left\langle\mathbf{i} \dot{a}^{0,1}, \bar{\beta} \alpha\right\rangle d v_{h}=0, \quad \forall \dot{a} .
$$

Hence $\bar{\beta} \alpha \equiv 0$. Since $\beta$ is a nontrivial holomorphic section its zero set is finite so that $\alpha \equiv 0$.

- $\dot{\beta}=\beta, \dot{a}=0$. We get

$$
\int_{\Sigma} f|\beta|^{2} d v_{h}=0
$$

Since $f$ is constant $(d f=0)$ the above equality implies $f \equiv 0$. This shows $\operatorname{ker} \Upsilon^{*}=0$.

Part (c) of the lemma now follows from part (b) coupled with (4.15).

Corollary 4.5. $H^{1}\left(V_{\beta, A}\right) \cong \operatorname{ker} H_{\beta, A}$ where

$$
\begin{aligned}
H_{\beta, A}=\Upsilon_{\beta, A} \oplus I_{\beta, A}^{*}: \Gamma\left(L \otimes K^{1 / 2}\right) \oplus \mathrm{i} \Omega^{1}(\Sigma) \\
\rightarrow\left(\Gamma\left(L \otimes K^{-1 / 2}\right) \oplus \mathrm{i} \Omega^{0}(\Sigma)\right) \bigoplus \mathrm{i} \Omega^{0}(\Sigma)
\end{aligned}
$$


is defined by

$$
H\left[\begin{array}{c}
\dot{\beta} \\
\mathbf{i} \dot{a}
\end{array}\right]=\left[\begin{array}{c}
\bar{\partial}_{A} \dot{\beta}+2^{-1 / 2} \mathbf{i} \dot{a}^{0,1} \beta \\
\mathbf{i} * d \dot{a}-\mathfrak{i} \mathfrak{i} \mathfrak{e}\langle\beta, \dot{\beta}\rangle \\
\mathbf{i} d^{*} \dot{a}-\mathbf{i} \mathfrak{I} \mathfrak{m}\langle\dot{\beta}, \beta\rangle
\end{array}\right]
$$

Denote by $\mathfrak{V}_{+}(L, \Sigma)$ the space of holomorphic $L$-valued vortices (eq. (4.14) ) and by $\mathfrak{V}_{+}^{*}$ the irreducible part

$$
\mathfrak{V}_{+}^{*}=\left\{(\beta, A) \in \mathfrak{V}_{+} ; \beta \neq 0\right\}
$$

Then the above lemma shows that if nonempty $\mathfrak{M}_{L}(\Sigma):=\mathfrak{V}_{+}^{*}(L) / \operatorname{Aut}(L)$ is a smooth manifold of dimension $2 d_{+}(L)$. Note that if $\operatorname{deg} L \neq 0$ then $\mathfrak{V}_{+}=\mathfrak{V}_{+}^{*}$. Moreover, the results of $[\mathrm{Br}]$ show that this moduli space is indeed nonempty and can be identified with the space of effective divisors on $\Sigma$ of degree $d_{+}(L)$. This space of divisors can then be identified with a symmetric product of $d_{+}(L)$ copies of $\Sigma$.

When $0 \leq \operatorname{deg} L \leq(g-1)$ one can construct similarly the space of $\mathfrak{V}_{-}(L)$ of antiholomorphic $L$-valued vortices. Its $\operatorname{Aut}(L)$-quotient will be a smooth manifold of dimension $2 d_{-}(L):=2(g-1)-2 \operatorname{deg} L$ (again a symmetric product).

Finally, define $\mathfrak{V}(L, \Sigma)=\mathfrak{V}_{\epsilon}(L)$, where $0<|\operatorname{deg} L| \leq(g-1)$ and $\epsilon=-\operatorname{sign} \operatorname{deg} L$.

With these 2-dimensional clarifications in place we can now return to our original 3-dimensional problem. We have seen that if $\pi: L_{N} \rightarrow N$ is a complex line bundle over $N$ there exist finitely many line bundles $L_{i} \rightarrow \Sigma$ over the base of $N$ such that

(i) $\left|\operatorname{deg} L_{i}\right| \leq(g-1)$ and

(ii) $L_{N} \cong \pi^{*}\left(L_{i}\right), \forall i$.

Denote by $\mathcal{C}^{*}\left(L_{i}\right)$ the space of irreducible configurations for the $L_{i}$-vortex problem on $\Sigma$ and by $\mathcal{C}_{N}^{*}$, the space of irreducible configurations for the Seiberg-Witten equations. Then we have the equalities

$$
\nabla \mathfrak{E}^{-1}(0)=\mathfrak{G}_{N} \cdot \bigcup_{i} \pi^{*} \mathfrak{V}\left(L_{i}\right)
$$

The adiabatic moduli space can then be represented as

$$
\nabla \mathfrak{E}^{-1}(0)=\left(\mathfrak{G}_{N} \cdot \bigcup_{i} \pi^{*} \mathfrak{V}\left(L_{i}\right)\right) / \mathfrak{G}_{N}
$$


The unrestricted moduli space

$$
\mathfrak{G}_{N} \cdot \bigcup_{i} \pi^{*} \mathfrak{V}^{1,2}\left(L_{i}\right)
$$

(equipped with the $L^{1,2}$ topology) is a $\mathfrak{G}_{N}$-invariant Hilbert manifold. This follows from the following simple observations.

(a) The first observation has an infinitesimal nature, namely if for some $(\phi, A) \in \pi^{*} \mathfrak{V}$ and some the infinitesimal gauge transformation if $\in \mathfrak{g}$ the induced tangent vector $\mathcal{L}_{\phi, A}(\mathbf{i} f)=(\mathbf{i} f \phi,-\mathbf{i} d f)$ on $\mathcal{C}_{N}$ is actually tangent to $\mathfrak{V}$ then if must be the pullback of some infinitesimal gauge transformation on $\Sigma$.

(b) The second observation has a global character namely that the stabilizer of each component $\pi^{*} \mathfrak{V}\left(L_{i}\right)$ coincides with the pulled-back gauge group $\pi^{*} \mathfrak{G}_{\Sigma}$.

The details can be safely left to the reader. We can now conclude that the quotient $\mathcal{A}_{\infty}$ can be given a smooth structure. Moreover, the tangent spaces can be identified with a cohomology group $H^{1}\left(V_{\bullet, \bullet}\right)$ arising from a suitable deformation complex.

\subsection{Bott nondegeneracy.}

We now have all the informations we need to prove Theorem 4.1. For applications to Floer theory it will be convenient to prove a sharper version of this theorem. We assume the base of $\pi: N \rightarrow \Sigma$ has genus $g \geq 2$.

Consider first a vortex pair $\left(\phi_{\Sigma}, A_{\Sigma}\right)$ on the base $\Sigma$ and we assume it is a holomorphic $L_{\Sigma}$-valued vortex $\phi_{\Sigma}=0 \oplus \beta_{\Sigma}$, where $L_{\Sigma} \rightarrow \Sigma$ is a complex line bundle such that $-(g-1) \leq \operatorname{deg} L<0$ and $\beta_{\Sigma}$ is a section of $L_{\Sigma} \otimes K_{\Sigma}^{1 / 2}$. Set $L=\pi^{*} L_{\Sigma}$. By pull-back we get a solution $(\phi, A)$ of $\left(S W_{\infty}\right)$ where $A=\pi^{*} A_{\Sigma}$ and $\phi=(0 \oplus \beta)=0 \oplus \pi^{*} \beta_{\Sigma}$.

According to Remark 3.7 the configuration $(\phi, A)$ is also a solution of the equation $\left(S W_{\infty}\right)_{\delta}$. We denote by $\hat{\mathfrak{H}}_{\delta}$ the stabilized hessian of $\mathfrak{E}_{\delta}$ at $(\phi, A)$. This is an unbounded, Fredholm, selfadjoint operator $\hat{\mathfrak{H}}_{\delta}: \mathfrak{Z}_{\delta} \rightarrow \mathfrak{Z}_{\delta}$. For each $\gamma \geq 0$ define $E_{\delta}(\gamma)$ as the space spanned by the $\mu$-eigenvectors corresponding to values $|\mu| \leq \gamma$. Since $\hat{\mathfrak{H}}_{\delta}$ is Fredholm this space is finite dimensional. Finally, define $E_{\infty}:=\operatorname{ker} H_{\beta, A}$. Via the pull-back map we can regard $E_{\infty}$ as a subspace of $T_{\phi, A} \mathcal{C} \subset \mathfrak{Z}$. More precisely, the following is true. 


\section{Proposition 4.6.}

$$
E_{\infty} \subset E_{\delta}(0), \quad \forall \delta>1
$$

Proof. Consider

$$
(\dot{\beta}, \dot{a}) \in \operatorname{ker} H_{\beta, A} \subset C^{\infty}\left(K^{1 / 2} \otimes L_{\Sigma}\right) \oplus \mathbf{i} \Omega^{1}(\Sigma) \stackrel{\pi^{*}}{\hookrightarrow} C^{\infty}\left(\mathcal{K}^{1 / 2} \otimes L\right) \oplus \mathbf{i} \Omega^{1}(N) .
$$

This means

$$
\left\{\begin{array}{r}
\bar{\partial}_{A} \dot{\beta}+2^{-1 / 2} \mathbf{i} \dot{a}^{0,1} \beta=0 \\
\mathrm{i} *_{\Sigma} d \dot{a}-\mathrm{i} \mathfrak{R e}\langle\beta, \dot{\beta}\rangle=0 \\
\mathbf{i} d^{*} \dot{a}-\mathrm{i} \mathfrak{I m}\langle\dot{\beta}, \beta\rangle=0
\end{array}\right.
$$

We claim that $(0 \oplus \dot{\beta}, \dot{a}, 0) \in \operatorname{ker} \hat{\mathfrak{H}}_{\phi, A}$ i.e. (conf. (4.6) with $f=0$ and $\left.\mathbf{D}_{A}=\mathfrak{D}_{A}-\lambda / 2\right)$

$$
\left\{\begin{array}{r}
\mathrm{D}_{A} \dot{\psi}+\mathbf{c}(\mathbf{i} \dot{a}) \phi=0 \\
-\mathbf{i} *_{N} d \dot{a}+\dot{\tau}(\phi, \dot{\psi})=0 \\
d^{*} \dot{a}-\mathfrak{I m}\langle\dot{\psi}, \phi\rangle=0
\end{array}\right.
$$

where $\dot{\psi}=0 \oplus \dot{\beta}$. This follows immediately from the following elementary observations.

- Since $\dot{a}$ is a pullback it is a basic invariant form i.e.

$$
L_{\zeta} \dot{a}=0, \quad \dot{a}(\zeta)=0 .
$$

This essentially shows that the last two equalities in (4.19) imply the last two equalities in (4.20).

- Since $\dot{\psi}$ is a pullback and so are the connections $A$ and $\nabla^{\infty}$ we deduce that $Z_{A, \infty} \dot{\psi}=0$. Using the identity $\mathbf{D}_{A}=Z_{A, \infty}+T_{A}$ it is not difficult to see that the first equality in (4.19) implies the first equality in (4.20). We leave the reader fill-in the missing details.

Our next result is a considerable improvement of the above proposition. 
Theorem 4.7. Consider a sequence $\gamma_{\delta}$ of nonnegative numbers such that

$$
\gamma_{\delta}=o(1) \text { as } \delta \rightarrow \infty
$$

Then

$$
E_{\delta}\left(\gamma_{\delta}\right)=E_{\infty}, \quad \forall \delta \gg 1
$$

Proof. We will argue by contradiction. Consider a sequence

$$
\Xi_{\delta}=\left(\dot{\psi}_{\delta}, \mathbf{i} \dot{a}_{\delta}, \mathbf{i} f_{\delta}\right) \in \mathfrak{Z} \delta
$$

of $\mu_{\delta}$-eigenvalues of $\hat{\mathfrak{H}}_{\phi, A, \delta}$ such that $\left|\mu_{\delta}\right| \leq \gamma_{\delta}$

$$
\begin{gathered}
\Xi_{\delta} \perp E_{\infty}(0) . \\
\left\|\Xi_{\delta}\right\|=\left\|\dot{\psi}_{\delta}\right\|_{2}+\left\|\omega_{\delta}\right\|_{2}+\left\|f_{\delta}\right\|_{2}=1, \quad \forall \delta \geq 1
\end{gathered}
$$

The $L^{2}$-norms are defined in terms of the metric $g . \omega_{\delta}$ is defined by $\omega_{\delta}=$ $\delta \dot{a}_{\delta}^{v} \eta+\dot{a}_{\delta}^{\perp}$ where $\dot{a}_{\delta}^{v}$ and $\dot{a}_{\delta}^{\perp}$ are given by

$$
\dot{a}_{\delta}^{v}=\dot{a}_{\delta}(\zeta), \quad \dot{a}_{\delta}^{\perp}=\dot{a}_{\delta}-\dot{a}_{\delta}^{v} \eta .
$$

Note that

$$
\delta^{1 / 2}\left\|\dot{a}_{\delta}^{v}\right\|_{2}+\left\|\dot{a}_{\delta}^{\perp}\right\|_{2}=\delta^{1 / 2}\left\|\dot{a}_{\delta}\right\|_{2, \delta}=\left\|\omega_{\delta}\right\|_{2}
$$

and

$$
\mathbf{c}_{\delta}\left(\dot{a}_{\delta}\right)=\mathbf{c}\left(\omega_{\delta}\right) .
$$

We will show that (on a subsequence) $\left\|\Xi_{\delta}\right\|=o(1)$ thus contradicting (4.22). The proof will be carried out in several steps. Decompose as usual $\dot{\psi}_{\delta}=$ $\dot{\alpha}_{\delta} \oplus \dot{\beta}_{\delta}$. Then

Step 1. $\left\|\dot{\alpha}_{\delta}\right\|_{1,2}=o(1)$ as $\delta \rightarrow \infty$ where the Sobolev norm is defined in terms of the metric $g$ and the fixed connection $A$.

Step 2. $\left\|f_{\delta}\right\|_{1,2}=O(1)$ and $\left\|\omega_{\delta}\right\|_{1,2}=O(1)$ as $\delta \rightarrow \infty$. (The above Sobolev norms are defined in terms of the metric $g$ and its Levi-Civita connec$\operatorname{tion}(\mathrm{s})$.)

Step 3. $Z_{A, \infty} \dot{\psi}_{\delta}=0, L_{\zeta} \dot{a}_{\delta}=0$ and $\partial_{\zeta} f_{\delta}=0$ for all $\delta \gg 1$.

Step 4. Conclusion. 
We now supply the details. Recall the notations $\mathbf{D}_{A, \delta}=\delta Z_{A, \infty}+T_{A}$ and $\hat{\nabla}^{A}$ - the connection on $\mathbb{S}_{L}$ obtained by tensoring the limiting Levi-Civita connection $\nabla^{\infty}$ on $\mathbb{S}$ with the connection $A$. The equations (4.6) can be rewritten as

$$
\begin{cases}\mathbf{D}_{A, \delta} \dot{\psi}_{\delta}+\left(\mathbf{c}_{\delta}\left(\mathbf{i} \dot{a}_{\delta}\right) \phi+\mathbf{i} f_{\delta}\right) \phi & =\mu_{\delta} \dot{\psi}_{\delta} \\ -\mathbf{i} *_{\delta} d \dot{a}_{\delta}+\dot{\tau}_{\delta}\left(\phi, \dot{\psi}_{\delta}\right)-\mathbf{i} d f_{\delta} & =\mu_{\delta} \mathbf{i} \dot{a}_{\delta} \\ d^{*} \dot{a}_{\delta} & =\mathfrak{I m}\left\langle\dot{\psi}_{\delta}, \phi\right\rangle\end{cases}
$$

For the proofs of steps 1-3 we only need to assume (4.22). The condition (4.21) will be used only at the final step.

Step 1. Set $\dot{\phi}=\dot{\phi}_{\delta}=\dot{\alpha}_{\delta} \oplus 0$ in the main variational identity (expressed in terms of the metric $g_{\delta}$ ). Using $d v_{\delta}=\delta^{-1} d v_{g}$ we get

$$
\begin{aligned}
& \text { (4.24) } \int_{N}\left|\mathbf{D}_{A, \delta} \dot{\phi}\right|^{2} d v_{g}+\int_{N}\left|\dot{\alpha}_{\delta}\right|^{2} \cdot|\phi|^{2} d v_{g} \\
& =\mathfrak{R e} \int_{N}\left\{\nu_{\delta}\left\langle\mathbf{c}_{\delta}\left(\mathbf{i} \dot{a}_{\delta}\right) \phi, \dot{\phi}\right\rangle+\left(\mu_{\delta}-\lambda_{\delta} / 2\right)\left\langle\mathbf{D}_{A, \delta} \dot{\phi}, \dot{\psi}_{\delta}\right\rangle+\frac{\lambda_{\delta} \mu_{\delta}}{2}\left|\dot{\alpha}_{\delta}\right|^{2}\right\} d v_{g}
\end{aligned}
$$

Neglecting the second term in the left-hand-side of the above equality we deduce

$$
\begin{aligned}
\left\|\mathbf{D}_{A, \delta} \dot{\phi}_{\delta}\right\|_{2}^{2} \leq & \left|\mu_{\delta}-\lambda_{\delta} / 2\right| \cdot\left\|\dot{\psi}_{\delta}\right\|_{2} \cdot\left\|\mathbf{D}_{A, \delta} \dot{\phi}_{\delta}\right\|_{2} \\
& +\left|\nu_{\delta}\right| \cdot\left\|\omega_{\delta}\right\|_{2} \cdot\|\phi\|_{\infty} \cdot\left\|\dot{\alpha}_{\delta}\right\|_{2}+\frac{\left|\lambda_{\delta} \mu_{\delta}\right|}{2}\left\|\dot{\alpha}_{\delta}\right\|_{2} .
\end{aligned}
$$

Thus, if we set $t_{\delta}=\left\|\mathbf{D}_{A, \delta} \dot{\phi}_{\delta}\right\|_{2}$ we obtain a quadratic inequality

$$
t_{\delta}^{2}=o\left(t_{\delta}+1\right)
$$

which implies $t_{\delta}=o(1)$ as $\delta \rightarrow \infty$. Using the adiabatic decoupling lemma we deduce that (on a subsequence) $\dot{\alpha}_{\delta} \oplus 0$ converges in $L^{1,2}$ to a spinor $\dot{\phi}_{\infty}=\dot{\alpha}_{\infty} \oplus 0$ satisfying $\mathbf{D}_{A} \dot{\phi}_{\infty}=0$. Using again (4.24) in which we neglect the first term in the left-hand-side we conclude that

$$
\int_{N}\left|\dot{\alpha}_{\delta}\right|^{2} \cdot|\phi|^{2} d v_{g}=o(1) \quad \text { as } \delta \rightarrow \infty .
$$

By passing to the limit we deduce

$$
\int_{N}\left|\dot{\phi}_{\infty}\right|^{2} \cdot|\phi|^{2} d v_{g}=0
$$


which implies $\dot{\alpha}_{\infty} \equiv 0$.

Step 2. Take the $g_{\delta}$-inner product with $d f_{\delta}$ of the second equation in (4.23). After an integration by parts we get

$$
\int_{N}\left|f_{\delta}\right|_{\delta}^{2} d v_{g}=\int_{N} g_{\delta}\left(d f_{\delta}, \mathfrak{I m} \dot{\tau}_{\delta}\left(\phi, \dot{\psi}_{\delta}\right)\right) d v_{g}
$$

Using the (pointwise) Cauchy-Schwartz inequality for the metric $g_{\delta}$ we deduce

$$
\int_{N} \delta^{2}\left|\partial_{\zeta} f_{\delta}\right|^{2}+\left|d f_{\delta}-\left(\partial_{\zeta} f_{\delta}\right) \eta\right|^{2} d v_{g} \int_{N}\left|d f_{\delta}\right|_{\delta}^{2} d v_{g}=O\left(\left\|\dot{\psi}_{\delta}\right\|_{2} \cdot \int_{N}\left|d f_{\delta}\right| d v_{g}\right) .
$$

This implies $\left\|d f_{\delta}\right\|_{2}=O(1)$ so that $\left\|f_{\delta}\right\|_{1,2}=O(1)$.

To establish the second estimate we first use Bochner's identity ([Ber])

$$
\Delta_{\delta} \dot{a}_{\delta}=\left(\nabla^{\delta}\right)^{*} \nabla \dot{a}_{\delta}+\operatorname{Ric}_{\delta} \dot{a}_{\delta}
$$

where $\Delta_{\delta}$ denotes the Hodge Laplacian (of $g_{\delta}$ ), $\nabla^{\delta}$ denotes the Levi-Civita connection of the metric $g_{\delta}$ and $\mathrm{Ric}_{\delta}$ denotes the Ricci curvature of the metric $g_{\delta}$. Since $\sup _{x \in N}\left|\operatorname{Ric}_{\delta}(x)\right|_{\delta}=O(1)$ the above inequality implies

$$
\int_{N}\left|\nabla^{\delta} \dot{a}_{\delta}\right|_{\delta}^{2} d v_{h}=O\left(\int_{N}\left|\dot{a}_{\delta}\right|_{\delta} d v_{g}\right)=O\left(\left\|\omega_{\delta}\right\|_{2}^{2}\right)=O(1) .
$$

Locally we have

$$
\left|\nabla^{\delta} \dot{a}_{\delta}\right|_{\delta}^{2}=\delta^{2}\left|\nabla_{\zeta}^{\delta} \dot{a}_{\delta}\right|_{\delta}+\left|\nabla_{\zeta_{1}}^{\delta} \dot{a}_{\delta}\right|_{\delta}^{2}+\left|\nabla_{\zeta_{2}}^{\delta} \dot{a}_{\delta}\right|_{\delta}^{2}
$$

On the other hand, since $\zeta$ is Killing we have

$$
\int_{N}\left|\nabla_{\zeta}^{\delta}\left(\dot{a}_{\delta}^{v} \eta+\dot{a}_{\delta}^{\perp}\right)\right|_{\delta}^{2} d v_{g}=\int_{N}\left|\nabla_{\zeta}^{\delta}\left(\dot{a}_{\delta}^{v} \eta\right)\right|_{\delta}^{2}+\left|\nabla_{\zeta}^{\delta} \dot{a}_{\delta}^{\perp}\right|_{\delta}^{2} d v_{g}
$$

which coupled with the above equality yields

$$
\int_{N}\left|\nabla^{\delta} \dot{a}_{\delta}\right|_{\delta}^{2} d v_{g} \geq \int_{N}\left|\nabla^{\delta} \omega_{\delta}\right| d v_{g}
$$

The structural equations of the metric $g_{\delta}$ imply immediately that

$$
\left\|\left(\nabla-\nabla^{\delta}\right) \omega_{\delta}\right\|_{2}=O\left(\left\|\omega_{\delta}\right\|\right)
$$


The equations (4.26)-(4.28) now imply that $\left\|\omega_{\delta}\right\|_{1,2}=O(1)$.

Step 3. This is essentially a "rigidity" result for the Hessian. More precisely, we will show that the natural $S^{1}$ action on $N$ induces an action on $\mathfrak{Z}_{\delta}$ such that $\hat{\mathfrak{H}}_{\phi, A, \delta}$ is $S^{1}$-equivariant. Thus the spectral spaces are $S^{1}$ modules and we will show that for $\delta \gg 1$ the weights of these modules are all trivial. The equalities at Step 2 are just infinitesimal reformulations of this fact but the proof we offer below does not explicitly mention the above representation theoretic remarks.

Define

$$
\Theta: C^{\infty}\left(\mathbb{S}_{L} \oplus T^{*} N \oplus \mathbb{R}\right) \rightarrow C^{\infty}\left(\mathbb{S}_{L} \oplus T^{*} N \oplus \mathbb{R}\right)
$$

by

$$
\Theta(\dot{\psi}, \dot{a}, f)=\mathbf{i}\left(\hat{\nabla}_{\zeta}^{A} \dot{\psi}, L_{\zeta} \dot{a}, \partial_{\zeta} f\right)
$$

Note first that

$$
\left[\hat{\mathfrak{H}}_{\phi, A, \delta}, \Theta\right]=0
$$

where $[\cdot, \cdot]$ denotes the commutator of two operators. The proof of (4.29) is a consequence of the following facts left to the reader as exercises.

- $\left[\hat{\nabla}_{\zeta}^{A}, \mathbf{D}_{A, \delta}\right]=0\left(\right.$ since $\left.\left\{Z_{A, \infty}, T_{A}\right\}=0\right)$

- $\left[\left(\hat{\nabla}_{\zeta}^{A}, \mathbf{c}_{\delta}(\dot{a})\right]=\mathbf{c}_{\delta}\left(L_{\zeta} \dot{a}\right)\right.$ (since the invariant $b(x)$ defined in (1.7) and (1.8) vanishes for the Boothby-Wang structures).

- $\left[L_{\zeta}, *_{\delta}\right]=0$ (since $\zeta$ is Killing).

- $\hat{\nabla}_{\zeta}^{A} \phi=0$ (since $Z_{A, \infty} \phi=0$.

- $\left[L_{\zeta}, d\right]=0$.

Since $\operatorname{div}_{g_{\delta}}(\zeta)=0$ the operator $\Theta^{2}$ is formally selfadjoint (with respect to the metric $\left.g_{\delta}\right)$ and from (4.29) we deduce that $\Theta^{2}$ is a non-negative, symmetric endomorphism of the spectral space $E_{\delta}\left(\gamma_{\delta}\right)$. Moreover, its eigenvalues can only be of the form $k^{2}, k \in \mathbb{Z}$ since only these can be eigenvalues of $-\partial_{\zeta}^{2}$ acting on $C^{\infty}(N)$, the third summand in the domain of $\Theta$. Assume now that $\forall \delta \gg 1$ the operator $\Theta^{2}$ acting on $E_{\delta}\left(\gamma_{\delta}\right)$ has a nontrivial eigenvalue $k_{\delta}^{2} \geq 1$. Assume now that $\Xi_{\delta}$ is a $k_{\delta}^{2}$-eigenvalue of $\Theta^{2}$. 
We now use the main variational identity with $\dot{\phi}=\dot{\psi}_{\delta}$, noting that in the term $\nabla_{\dot{a}} \phi$ the $\zeta$-component vanishes (since $Z_{A, \infty} \phi=0$ ). If we set $t_{\delta}=\left\|\mathbf{D}_{A, \delta} \dot{\psi}_{\delta}\right\|_{2}$ then we obtain as in the proof of Step 1

$$
t_{\delta}^{2} \leq C\left(\left\|\dot{\psi}_{\delta}\right\|_{2}+o\left(t_{\delta}\right)\right)
$$

which implies $t_{\delta}^{2}=O(1)$. On the other hand, $t_{\delta}^{2}=\delta^{2} k_{\delta}^{2}\left\|\dot{\psi}_{\delta}\right\|_{2}^{2}+\left\|T_{A} \dot{\psi}_{\delta}\right\|_{2}^{2}$ which shows that

$$
\left\|\dot{\psi}_{\delta}\right\|=o(1)
$$

Using this last information in the inequality (4.30) we conclude that $t_{\delta}^{2}=$ $o\left(1+t_{\delta}\right)$ which implies

$$
\left\|\mathrm{D}_{A, \delta} \dot{\psi}_{\delta}\right\|_{2}=o(1) .
$$

We can now choose a subsequence $\delta \rightarrow \infty$ such that

$$
\left(\dot{\psi}_{\delta}, \omega_{\delta}, f_{\delta}\right) \rightarrow\left(\dot{\psi}_{\infty}, \omega_{\infty}, f_{\infty}\right) \text { strongly in } L^{2}
$$

In particular, the equality (4.22) implies

$$
\left\|\dot{\psi}_{\infty}\right\|_{2}+\left\|\omega_{\infty}\right\|_{2}+\left\|f_{\infty}\right\|_{2}=1
$$

The inequality (4.31) implies $\dot{\psi}_{\infty} \equiv 0$.

On the other hand, the inequalities (4.25) and (4.31) yield

$$
\int_{N}\left|d f_{\delta}\right|_{\delta}^{2} d v_{g}=o(1)
$$

and in particular

$$
\delta^{2} k_{\delta}^{2}\left\|f_{\delta}\right\|_{2}^{2}=\int_{N}\left|\partial_{\zeta} f_{\delta}\right|^{2} \cdot|\eta|_{\delta}^{2} d v_{g}=o(1)
$$

so that

$$
\left\|f_{\delta}\right\|_{2}=o(1) \text {. }
$$

Hence $f_{\infty} \equiv 0$ as well. 
We now pass to the limit in the first equation of (4.23) using the inequality (4.32). We get

$$
\mathbf{c}\left(\mathbf{i} \omega_{\infty}\right) \phi=0 \text {. }
$$

Since $\phi^{-1}(0)$ is a finite set of fibers of $N$ we deduce $\omega_{\infty}=0$ almost everywhere. This contradicts (3.6) and concludes the proof of Step 3.

Step 4. Using the information from Step 3 we deduce that $\mathbf{D}_{A} \dot{\psi}_{\delta}=\mathbf{D}_{A, \delta} \dot{\psi}_{\delta}$ for all $\delta \gg 1$. The first equation of (4.23) coupled with (4.22) implies

$$
\left\|\mathbf{D}_{A} \dot{\psi}_{\delta}\right\|_{2}=O(1)
$$

Invoking the elliptic estimates for the operator $\mathbf{D}_{A}$ we deduce that $\left\|\dot{\psi}_{\delta}\right\|_{1,2}=$ $O(1)$. Pick now a subsequence $\delta \rightarrow \infty$ such that

$$
\left(\dot{\psi}_{\delta}, \omega_{\delta}, f_{\delta}\right) \rightarrow\left(\dot{\psi}_{\infty}, \omega_{\infty}, f_{\infty}\right) \text { in the norm of } L^{2} .
$$

From the equality

$$
\mathbf{D}_{A} \dot{\psi}_{\delta}+\left(\mathbf{c}\left(\mathbf{i} \omega_{\delta}\right)+\mathbf{i} f_{\delta}\right) \phi=\mu_{\delta} \dot{\psi}_{\delta}
$$

we deduce (via elliptic estimates again) that $\dot{\psi}_{\delta}$ is a Cauchy sequence in $L^{1,2}$ and thus converges strongly in this Banach space. We can now pass to the limit in the above equality using the fact that $Z_{A, \infty} \dot{\psi}_{\delta}=0$ and we deduce

$$
T_{A} \dot{\psi}_{\infty}+\left(\mathbf{c}\left(\mathbf{i} \omega_{\infty}\right)+\mathbf{i} f_{\infty}\right) \phi=0 .
$$

Using the decompositions $\dot{\psi}_{\infty}=0 \oplus \dot{\beta}_{\infty}$ (Step 1) and $\phi=0 \oplus \beta$ we deduce

$$
\left(\omega_{\infty}(\zeta)+\mathbf{i} f_{\infty}\right) \beta=0
$$

Since $\beta^{-1}(0)$ is a finite collections of fibers we deduce from the above equality that

$$
f_{\infty}=\omega_{\infty}(\zeta)=0 \text { a.e. on } N .
$$

In particular $\left\|\dot{a}_{\delta}\right\|_{2}+\delta\left\|\dot{a}_{\delta}^{v}\right\|_{2}=o(1)$ and $L_{\zeta} \omega_{\infty}=0$. Hence $\left(\dot{\psi}_{\infty}, \omega_{\infty}, f_{\infty}\right)$ is a pullback of a similar object on the base $\Sigma$. Using all the informations we have gathered up to this point it is a simple exercise (left to the reader) to show that $\Xi_{\infty}=\left(\dot{\psi}_{\infty}, \mathbf{i} \omega_{\infty}, 0\right) \in \mathfrak{Z}$ is a 0 -eigenvector of the extended $g$-Hessian i.e.

$$
\hat{\mathfrak{H}}_{\phi, A} \Xi_{\infty}=0 \text {. }
$$

Using the fact that $\Xi_{\infty}$ is a pullback we deduce $\Xi_{\infty} \in E_{\infty}(0)$. This contradicts the assumption (4.21). Theorem 4.7 is proved.

Theorem 4.7 can also be rephrased as a spectral estimate. 
Corollary 4.8. There exist constants $\gamma_{\infty}>0$ and $\delta_{\infty}>1$ such that for any irreducible adiabatic solution and any $\delta \geq \delta_{\infty}$ the only eigenvalue of $\mathfrak{H}_{\phi, A, \delta}$ in the interval $\left[-\gamma_{\infty}, \gamma_{\infty}\right]$ is 0 and moreover the corresponding eigenvectors span the space $E_{\infty}(0)$ which is the tangent space at $(\phi, A)$ of the adiabatic moduli space.

The spectral gap estimate can be used (in combination with the techniques of [T]) to "reverse" the adiabatic flow i.e. to reconstruct the solutions of the original equations starting from the adiabatic ones. This may not be a very useful thing to do since the adiabatic equations are better suited for topological computations due mainly to the fact that they can be solved explicitly. Since they are (zeroth order) perturbations of the original equations one will obtain the same results.

\section{References.}

[Ber] P.H. Berard, From vanishing theorems to estimating theorems: the Bochner technique revisited, Bull. A.M.S., 19 (1988), 371-406.

[BGV] N. Berline, E. Getzler and M. Vergne, Heat Kernels And Dirac Operators, Springer Verlag, 1992.

[BC] J.M. Bismut and J. Cheeger, $\eta$-invariants and their adiabatic limits, Journ. Amer. Math. Soc., 2 (1989), 33-70.

[B] D.E. Blair, Contact Manifolds In Riemann Geometry, Lecture Notes in Math 509, Springer Verlag, 1976.

[BW] W.M. Boothby and H.C. Wang, On contact manifolds, Ann. of Math. vol. 68(1958), 721-734.

[Br] S. Bradlow, Vortices in holomorphic line bundles over closed Kähler manifolds, Comm. Math. Phys., 135(1990), 1-17.

[BGP] S. Bradlow and O. Garcia-Prada, Non-abelian monopoles and vortices, alg-geom $/ 9602010$.

[CH] S.S. Chern and R.S. Hamilton, On Riemann metrics adapted to three dimensional contact manifolds, in the volume "Arbeitstagung Bonn: proceedings of the meeting held in Bonn, June 15-22, 1984", Lecture Notes in Mathematics, Springer-Verlag 1985, 279-305.

[Dai] X. Dai, Adiabatic limits, nonmultiplicativity of signature and Leray spectral sequence, J.A.M.S., 4(1991), 265-321. 
[D] S.K. Donaldson, The Seiberg-Witten equations and 4-manifold topology, B.A.M.S. vol.33 (1996), 45-70.

[ENS] F. Ehlers, W.D. Neumann and J. Scherk, Links of surface singularities and CR space forms, Comment. Math. Helvetici 62(1987), 240-264.

[FU] D.S. Freed and K.K. Uhlenbeck, Instantons and four-manifolds, MSRI Publications, vol. 1, Springer Verlag, New York, 1984.

[FS] M. Furuta and B. Steer, Seifert fibered homology spheres and the Yang-Mills equations on Riemann surfaces with marked points, Adv. in Math., 96(1992), 38-102.

[H] L. Hörmander, Uniqueness theorems for second order elliptic differential equations, Comm. Part. Diff. Eq. vol. 8 (1983), 21-64.

[JN] M. Jankins and W.D. Neumann, Lectures On Seifert Manifolds, Brandeis Lecture Notes, 1983.

[K] T. Kato, Perturbation Theory For Linear Operators, Springer Verlag, 1984.

[KM] P.B. Kronheimer and T.S. Mrowka, The genus of embedded surfaces in the projective plane, Math. Res. Letters 1 (1994), 797-808.

[LM] H.B. Lawson and M.L. Michelson, Spin Geometry, Princeton Mathematical Series 38, Princeton University Press, 1989.

[MD] I.G. MacDonald, Symmetric products of an algebraic curve, Topology, 1 (1962), 319-343.

[Mar] M. Marcolli, Seiberg-Witten Floer homology and Heegard splittings, dgga/9601011.

[MW] M. Marcolli and B. Wang, Equivariant Seiberg-Witten-Floer homology, dg-ga/9606003.

[MM] R. Mazzeo and R. Melrose, The adiabatic limit, Hodge cohomology and Leray's spectral sequence for a fibration, J. Diff. Geom., 31(1990), 183213.

[MST] J.W. Morgan, Z. Szabo and C.H. Taubes, A product formula for the Seiberg-Witten invariants and the generalized Thom conjecture, Journ. Diff. Geom., 44(1997), 706-788.

[MOY] T. Mrowka, P. Ozsvath and B. Yu, Seiberg-Witten monopoles on Seifert fibered spaces, MSRI preprint, 1996/93. 
[Mun] V. Munoz, Constraints for Seiberg-Witten basic classes of glued manifolds, dg-ga /9511012.

[Ne] W.D. Neumann, Geometry of quasihomogeneous surface singularities, Proc. Symp. Pure Math. AMS, 40(1983), v.2, 245-258.

[NR] W.D. Neumann and F. Raymond, Seifert manifolds, plumbing, $\mu$ invariant and orientation reversing maps, Proc. Alg, and Geom. Topology, (Santa Barbara 1977), Springer Lectures Notes 644, 1978.

[RV] F. Raymond and A.T. Vasquez, 3-manifolds whose universal coverings are Lie groups, Topology and its applications, 12 (1981), 161-179.

[Sas] S. Sasaki, On a property of Brieskorn manifolds, Tohoku Math. J., 28 (1976), 67-78.

[Sch] J. Scherk, CR structures on the link of an isolated singular point, Canadian Math. Soc., Conference Proc., vol. 6 (1986), 397-403.

[S] P. Scott, The geometries of 3-manifolds, Bull. London. Math. Soc., 15 (1983), 401-487.

[T] C.H. Taubes, Self-dual Yang Mills connections on 4-manifolds with indefinite intersection matrix, J. Diff. Geom., 19(1984), 517-560.

[V] A.N. Varchenko, Contact structures and isolated singularities, Vestnik Moscow Univ. Ser. I Mat. Mekh, 35 (1980), 18-21.

[Wa] B. Wang, Seiberg-Witten-Floer homology for homology 3-spheres, dgga/9602003.

[We] A. Weinstein, Three dimensional contact manifolds with vanishing torsion tensor, in the volume "Arbeitstagung Bonn: proceedings of the meeting held in Bonn, June 15-22, 1984", Lecture Notes in Mathematics, Springer-Verlag 1985, 306-308.

[Wo] J.A. Wolf, Spaces Of Constant Curvature, Publish or Perish, Boston, 1974.

[YK] K. Yano and M. Kon, Structures On Manifolds, Series in Pure Math. vol. 3, World Scientific, 1984.

ReCeIVEd DeCEMBER 16, 1996.

AdDRESS BEGINNING JULY 1997

MCMASTER UNIVERSITY

DEPARTMENT OF MATHEMATICS

Hamilton, Ontario, Canada, L8S 4K1 